\title{
Engineering on Anatomy to Avoid the Necessity of Invasive Imaging in Medical 3D Printing: Examples from Pediatric Cardiology
}

Carina Hopfner ( $\nabla$ carina.hopfner@med.uni-muenchen.de)

Klinikum der Universitat Munchen Standort Grosshadern https://orcid.org/0000-0002-2263-2477

Andre Jakob

Klinikum der Universitat Munchen Standort Grosshadern

Anja Ingrid Tengler

Klinikum der Universitat Munchen Standort Grosshadern

Maximilian Grab

Klinikum der Universitat Munchen Standort Grosshadern

\section{Nikolaus Thierfelder}

Klinikum der Universitat Munchen Standort Grosshadern

\section{Barbara Brunner}

Klinikum der Universitat Munchen Standort Grosshadern

Alisa Thierij

Klinikum der Universitat Munchen Standort Grosshadern

Nikolaus Haas

Klinikum der Universitat Munchen Standort Grosshadern

\section{Technical Note}

Keywords: 3D Printing in Medicine, Non-patient-specific 3D Modeling, Simulation Training, Pediatric Cardiology, 3D Heart Models, Pulsatile Heart Model

Posted Date: August 3rd, 2020

DOl: https://doi.org/10.21203/rs.3.rs-51075/v1

License: (c) (i) This work is licensed under a Creative Commons Attribution 4.0 International License. Read Full License 


\section{Engineering on Anatomy to Avoid the Necessity of Invasive Imaging in Medical 3D Printing: Examples from Pediatric Cardiology}

Carina Hopfner ${ }^{1}$, Andre Jakob ${ }^{1}$, Anja Tengler ${ }^{1}$, Maximilian Grab², Nikolaus Thierfelder ${ }^{2}$, Barbara Brunner $^{1}$, Alisa Thierij ${ }^{1}$, Nikolaus A. Haas ${ }^{1}$

Corresponding author: $\quad$ Carina Hopfner, M.Sc. ${ }^{1}$

Co-authors: $\quad$ Andre Jakob, $M^{1}$

Anja Tengler, $\mathrm{MD}^{1}$

Maximilian Grab, M.Sc. ${ }^{2}$

Nikolaus Thierfelder, $\mathrm{MD}^{2}$

Barbara Brunner, cand.med. ${ }^{1}$

Alisa Thierij, cand.med. ${ }^{1}$

Nikolaus A. Haas, MD, $\mathrm{PhD}^{1}$

Affiliations: $\quad$ 'LMU Klinikum, Department for Pediatric Cardiology and Intensive Care, Munich, Germany;

${ }^{2}$ LMU Klinikum, Clinic and Policlinic for Heart Surgery, Munich, Germany

Address for correspondence:

Carina Hopfner, M.Sc.

LMU Klinikum, Campus Großhadern

Department for Pediatric Cardiology and Intensive Care

Marchioninistr. 15

D-81377 Munich

Germany

Phone: +49 $894400-76942$

Fax: $+49894400-73943$

carina.hopfner@med.uni-muenchen.de 

submission. The content of the manuscript has not been published or submitted for publication elsewhere.

The manuscript is submitted to the thematic series of "Advanced Image Segmentation and Modeling for 3D Printing in Medicine".

3D printed models based on CT or MRI datasets of clinical patients are often used for educational and training purposes. For these applications a patient-specific representation of the anatomy is not always necessary. Instead, existing datasets or 3D models can be digitally modified by advanced segmentation and design techniques to create models with variant anatomical and pathological findings. This approach avoids the necessity of invasive imaging and allows the creation of numerous different anatomical models individually tailored to their intended training purpose. This makes it a relevant topic for the 3D Printing in Medicine journal and its community.

Key words:

3D Printing in Medicine, Non-patient-specific 3D Modeling,

Simulation Training, Pediatric Cardiology, 3D Heart Models, Pulsatile Heart Model

Word count abstract: 393

Word count: 


\section{Abstract}

Background: 3D printed models of pediatric hearts with congenital heart disease (CHD) have been proven helpful in simulation training of diagnostic and interventional catheterization. However, anatomically accurate 3D printed models are traditionally based on real 2D scans of patients requiring specific imaging techniques, i.e. computer tomography (CT) or magnetic resonance imaging (MRI). In small children both imaging technologies often require deep sedation and involve radiation (CT) being of special impact in this population. Hence, cardiac image data acquired by these invasive technologies is rare in pediatrics where minimization of radiation and sedation is key. Therefore, an alternative solution to create variant 3D printed heart models for teaching and hands-on training has been established.

Methods: In this study different methods utilizing image processing and computer aided design (software: Mimics Innovation Suite, Materialise NV) have been established to overcome this shortage and to allow unlimited variations of 3D heart models based on a single patient scan. Patient-specific models based on a CT or MRI image stack were modified by performing virtual engineering on the original shape and structure of the heart. Thereby, 3D hearts including several pathological findings were created and CHD training models were adapted to training level and aims of hands-on classes, particularly for invasive procedures such as interventional cardiology.

Results: By changing the shape and structure of the 3D anatomy various training models were created of which four examples are presented in this paper: 1. a heart model with a patent ductus arteriosus (PDA) augmented by perimembranous ventricular septal defect (pmVSD) and muscular ventricular septal defect (mVSD), 2. a model of solely the right heart with pulmonary valve stenosis (PS) augmented by the left heart and myocardium, 3. a series of heart models with atrial septal defect (ASD) 
showing the hemodynamic effect on the right atrial and ventricular wall, 4 . a model of solely the left heart with isthmus stenosis augmented with an engineered aortic valve. All presented models have been successfully utilized in teaching or hands-on training courses.

Conclusions: It has been demonstrated that structure and shape of 3D heart models can be modified virtually by engineering on anatomy. Therefore, anatomical variants can be created without the necessity for real, patient-specific CT or MRI imaging. Further investigations are required to evaluate the resemblance of reality of nonpatient-specific 3D models and to prove the effectiveness of training using these designed heart models. 


\section{Background}

Simulation training has been established in professions associated with high-risk and considerable demands on knowledge and skills. The best known example is the use of a flight simulator in the education and training in aviation (1). This concept has been transferred to the medical field and has become the gold standard for resuscitation training. The training courses regulated by international guidelines include Immediate Life Support (ILS), Advanced Life Support (ALS), Neonatal Life Support (NLS), European Pediatric Immediate Life Support (EPILS) and European Pediatric Advanced Live Support (EPALS). The basic courses are recommended for the general public, i.e. also non-physicians, and mandatory for medical personal. Advanced courses are intended specifically for medical professionals in both, pediatric and adult medicine, involved in resuscitation teams. (2) There are other medical fields utilizing the concept of simulation in education and training. For example, applications of 3D printed models for training have been described for neurosurgery, otolaryngology, cardiac surgery and adult cardiology. $(1,3)$ However, in pediatric cardiology the standard methodology for education still follows the apprenticeship-model, i.e. the trainee observes, takes over step-by-step and practices under supervision until he or she can act independently. (4) As of today, there are no simulation training setups available today for the hands-on practice of diagnostic and interventional catheterization in congenital heart disease (CHD).

Therefore, a simulation training based on 3D printed heart models has been established in this study. Image processing and additive manufacturing technologies enable the creation of 3D models based on computer tomography (CT) or magnetic resonance imaging (MRI) scans and subsequently the printing of realistic threedimensional replicas of human hearts. 3D printed heart models are suitable for 
morphology courses (5-12), education of nurses (13), surgical training (14-20), surgical planning (3) and patient education (21-23). The cardiac 3D replicas produced in this study have been successfully utilized in hands-on catheterization courses for students, residents and experienced cardiologists. The setup of the simulation training allows training of the complete procedure of diagnostics and interventional treatment for both roles, first and second operator respectively. Since the flexible printing material (3D printer: Agilista-3200W, Keyence Corporation) used for the 3D heart models is radiopaque it can be examined via X-ray imaging. It is hence ideally suitable for handson catheter trainings $(24,25)$.

Various studies have proven the effectiveness of simulator training in medicine and shown that the request and need for 3D printed models in medical education increases continuously. $(1,26)$ Today, 3D printed heart models are generated from CT and MRI scans of specific patients. These imaging techniques involve harmful effects for patients, such as radiation for CT and often sedation of young children for the conduct of MRI. In the case of ultrasound imaging being able to provide the same information as a CT or MRI scan can, it is the imaging technique of choice for pediatric patients. (27) Therefore, the acquisition of $2 \mathrm{D}$ image stacks, such as CT and MRI scans, of patients with CHD are not always indicated and rare.

The aim of this study is to demonstrate the possibility of creating variant 3D printed CHD models by modifying existing patient-specific heart models instead of using CT or MRI data according to the most common anatomical variations. Image processing software was used that offers the possibility to segment 2D image stacks and convert them to $3 \mathrm{D}$ virtual surface models. Additionally, it provides a variety of tools allowing engineering on the $3 D$ anatomy to create new versions of $3 D$ hearts. Some of the 
techniques and selected examples of 3D printed CHD models are presented in the following.

\section{Methods}

3D models of patients' anatomy can be reconstructed from 2D image stacks acquired by $\mathrm{CT}$ or MRI. Image processing software is thereby used for the segmentation of $2 \mathrm{D}$ data and its transfer to a 3D STL file. STL is a standard file format for computer aided design (CAD) and 3D printing software. This three-dimensional surface object can then be further adapted in its size and shape with the possibility of designing anatomical variations based on a single patient scan. In this report the Materialise Mimics Innovation Suite (Materialise NV) was used for segmentation (Materialise Mimics 22.0) and design (Materialise 3-matic 14.0) of 3D anatomical models. In the following, the process of creating a patient-specific $3 \mathrm{D}$ model based on a $2 \mathrm{D}$ image stack is described as well as the techniques applied for engineering on anatomy to create 3D models of anatomical variety all based on the same scan.

\section{Patient-specific 3D heart models:}

Creating a 3D heart model based on a CT or MRI image stack starts by segmentation of the blood volume depicted in the $2 \mathrm{D}$ patient scan. In general, the blood volume is clearly visible due to the contrast fluid used for imaging. First all pixels with grey values within the threshold range of contrast enhanced blood are marked and saved as a region of interest $(\mathrm{ROI})$ which consists of $3 \mathrm{D}$ voxels built up by the pixels marked in subsequent 2D slices. Structures within the same threshold range unrelated to the marked cardiovascular structures (e.g. bone) can be deleted from the ROI by seed point methods or unmarking pixels. These techniques limit the ROI to the structures supposed to be represented in the final 3D heart model. Examples are the separation 
of the left and right heart or the shortening of long vessels. For some image datasets these first basic segmentation steps already result in an anatomically correct representation of the inner volume of the heart. However, in most cases poor image resolution, inhomogeneous distribution of contrast agent or metal artefacts will require more advanced segmentation techniques and skills. The key image processing methods in the handling of said issues are local thresholding of a defined limited area, application of filling algorithms involving edge detection and the use of Boolean operations applied to ROls with different threshold ranges. Local thresholding and filling are particularly helpful for the adaptation of regions with less or scattered contrast fluid. Boolean operations are utilized to manage metal artefacts. An overview of the imaging issues and image processing solutions is illustrated in figure 1.

\section{Common Imaging Issues}

- poor image resolution

- inhomogeneous distribution of contrast agent

- metal artefacts

\section{Image Processing Solutions}

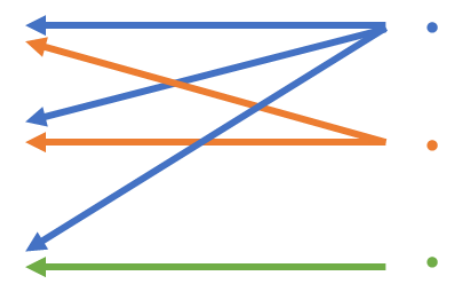

local thresholding of a defined limited area filling algorithms involving edge detection

Boolean operations applied to ROIs with different threshold ranges

FIGURE 1: Various image processing solutions are provided to overcome imaging issues. Boolean operations between ROls with different threshold ranges support the segmentation of datasets with metal artefacts. Filling algorithms involving edge detection are helpful for images with poor resolution or inhomogeneously distributed contrast agent. Local thresholding is utilized for advanced segmentation in all said cases. 
Once the selected ROI of the blood volume includes all structures of the heart desired to be represented in the final 3D heart model, an STL file is calculated. Thereby, the 3D volume consisting of voxels is translated into a surface model defined by triangles. The software Materialise Mimics then visualizes the contour of the calculated surface model in the $2 \mathrm{D}$ images enabling the control of the resemblance of reality of the engineered structure. The process is depicted in figure 2.
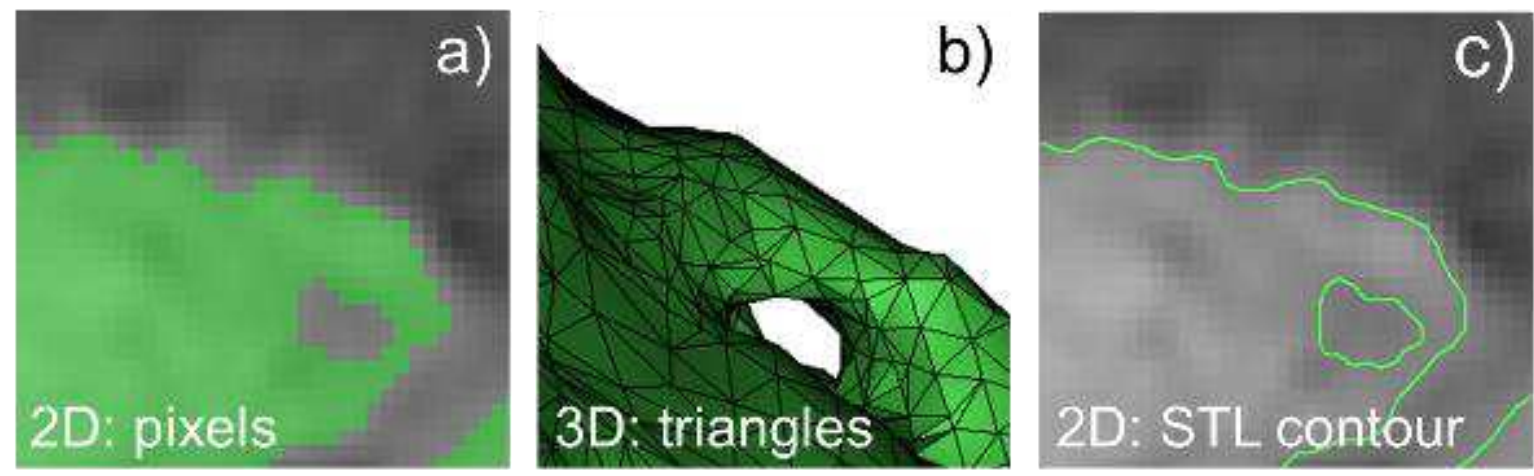

FIGURE 2: a) Pixels marked in green on one slice of a 2D image stack. Marked pixels on subsequent slices build up voxels in 3D. b) STL surface file calculated from those voxels. The surface is defined by triangles. c) The contour of the STL can be visualized in the 2D images for control of the resemblance of reality.

The calculation of the STL file is the basis for further design adaptations regarding size and shape of the heart model. It can be edited in both software parts of the Mimics Innovation Suite, the segmentation software Mimics and the design software 3-matic. The advantage of working in Mimics is that changes to the model surface can be compared to the original patient data by observing the contour of the STL in the images. Alternatively, a model edited in 3-matic can be transferred back to Mimics for observation and control of the contour. 
First design operations are dedicated to improving the surface of the virtual copy of the blood volume. These tools include smoothing, closing undesired small holes and deleting floating shells inside the model which occur due to inhomogeneous distribution of contrast fluid (unmarked areas within the ROI). The operations can be executed globally or locally on the model. The results can be monitored by comparing the resulting surface contour with the actual pixels in the 2D images.

The optimized STL of the intracardiac surface is then used to create a hollow heart model. Therefore, a second surface wrapped around the outside of the blood volume is calculated building a wall of user-defined thickness. The wall thickness can be chosen individually and mostly depends on the choice of 3D printing technology and material used as well as the application of the 3D model. To access the interior of the printed 3D model, openings have to be created virtually on the STL by cutting open the vessel endings. These openings also serve to remove support structures required for the 3D printing process.

\section{Non-patient-specific "engineered" 3D heart models:}

More advanced computer aided 3D design techniques for STLs are available which are mostly intended for use in industrial design, the original application for $C A D$ software. However, these software functions can also be applied to medical 3D models to create a variety of anatomical shapes and characteristics.

The most simple but also important operation on 3D models is scaling. With regards to training models this tool offers the possibility of creating heart models adapted to any patient size or age from infant to adult. By just scaling the artificial heart one dataset can already cover a patient spectrum with the same disease but of different size. This impacts the creation of training models as the size of the heart - natural or 3D printed 
- is a central aspect affecting the degree of difficulty in the performance of catheterization. Thus, trainees can start practicing a procedure on an adult size heart and slowly migrate to smaller versions of the same heart with increasing requirements to their dexterity but concentrating on one disease and procedure (figure 3).
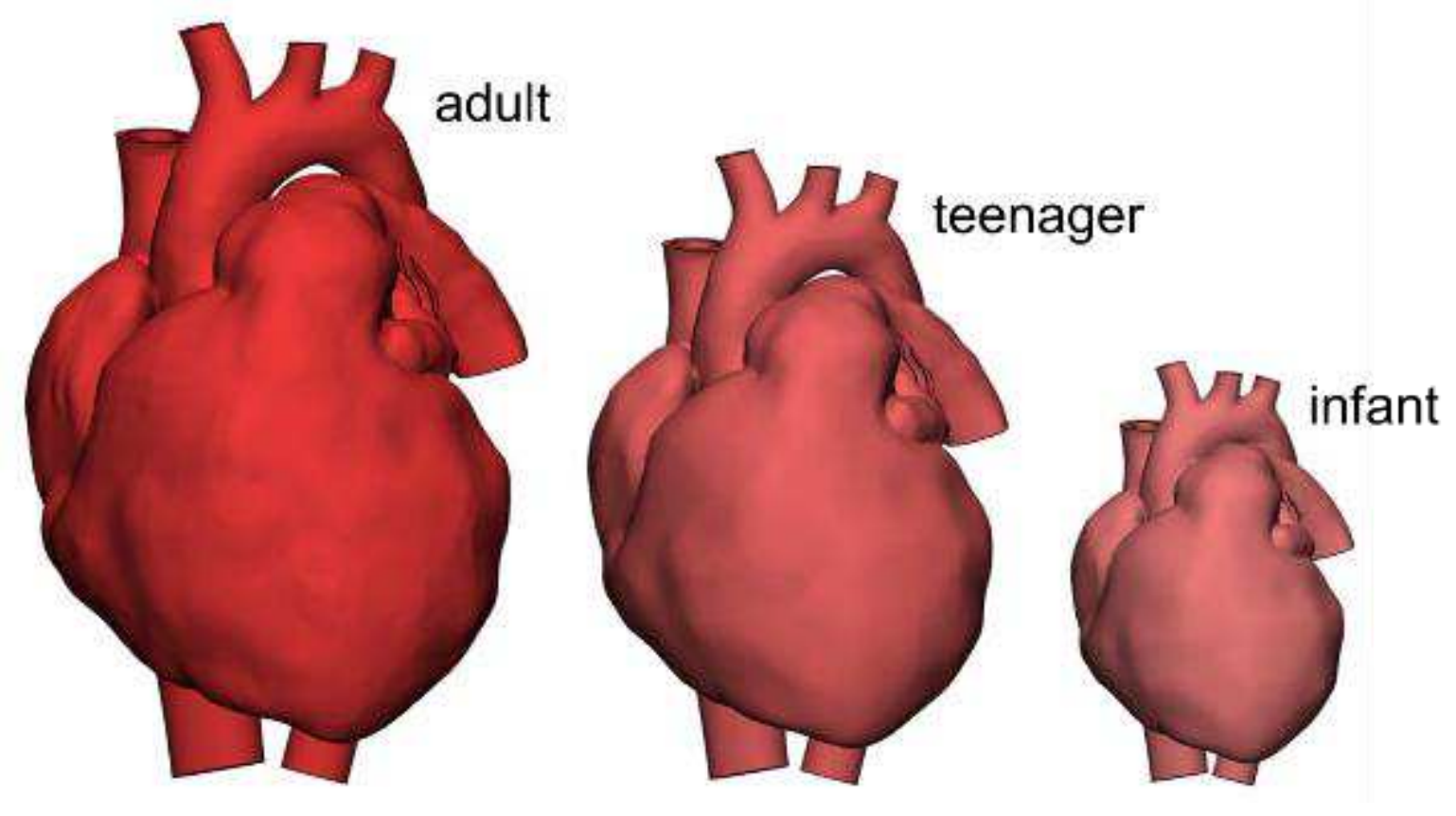

FIGURE 3: 3D heart models of different sizes. The adult sized heart was created based on a CT scan. For the teenager and infant hearts the original model was scaled to enlarge the patient spectrum depicted in training models. Therefore, three different complexity levels for training were generated from a single scan of a patient.

For insight into the model and access to the interior for training purposes, but also to remove support structures generated during the 3D printing process it is important to include windows or other openings in the model design. Therefore, trimming vessel endings, parts or whole structures of the 3D heart is one option. This technique is especially applicable for morphology teaching models as it allows wide opening and therefore clear exposure of anatomical structures inside the heart. For models 
requiring smaller, more geometric openings, windows of any shape and size can be created. Open windows in the model wall can be generated by Boolean subtraction of a cylinder or other 3D shapes (e.g. cone, free form, etc.). Flap-shaped windows, giving the model a closed appearance, can be integrated using different approaches, e.g. extruding the sketch of the window outline. The afore mentioned examples of openings for 3D hollow heart models are depicted in figure 4.
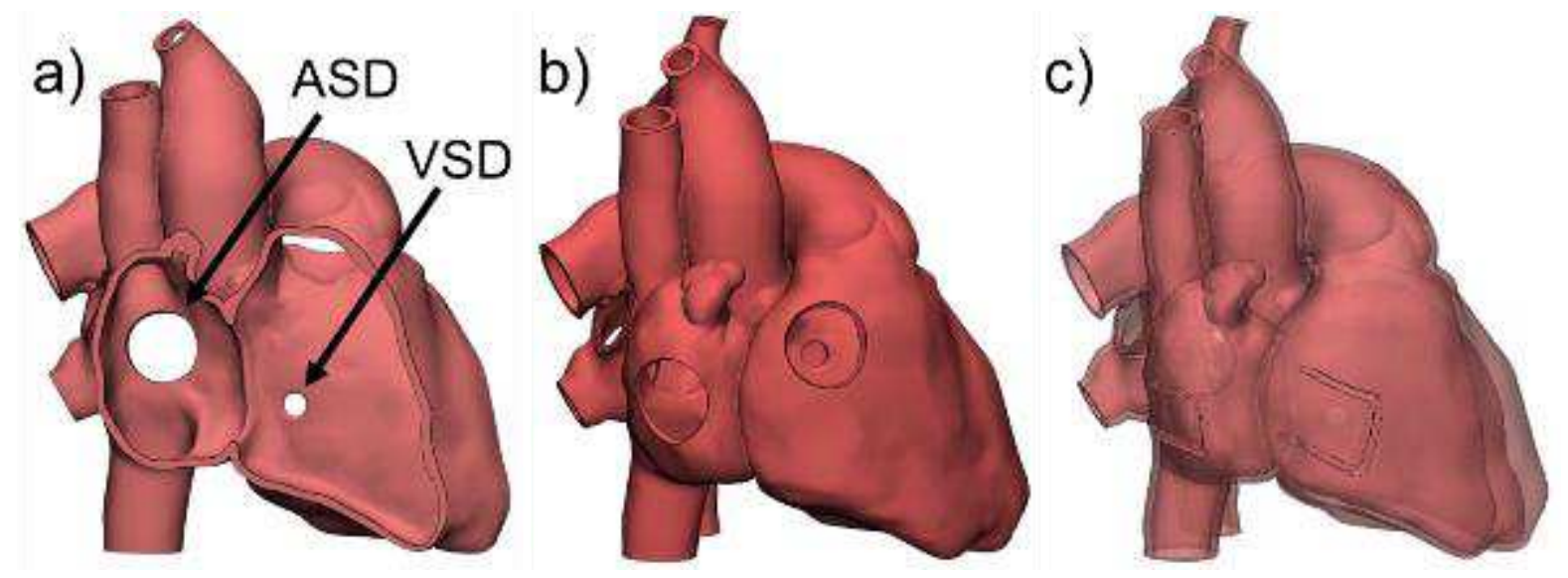

FIGURE 4: The vessels of all depicted 3D models have been opened by trimming operations on the respective STL. a) Cutting off larger parts and whole structures of the heart allows clear visualization of anatomical structure, in this case the septum and septal defects (atrial septal defect (ASD), ventricular septal defect (VSD)). The remaining parts of the ventricles can be either omitted or equipped with sockets for magnets to create an assembly model after 3D printing. b) Circular windows allow insight into intracardiac structures, but at the same time maintain a closed shape of the heart. c) Windows designed as flaps are appropriate particularly for models printed in flexible material. The appearance of heart models with flap-windows is even more closed and complete.

The closure of a natural anatomical hole (e.g. atrial septal defect (ASD), ventricular septal defect (VSD)) or the connection between structures (e.g. patent ductus 
arteriosus (PDA)) can also be of Interest. Engineering on STLs allows the manipulation of the surface structure so that surface areas can be marked, deleted and replaced by new surfaces. Further post-processing by smoothing and rearranging the STL triangles within the treated area makes the design changes invisible. Figure 5 pictures the closure of a VSD.
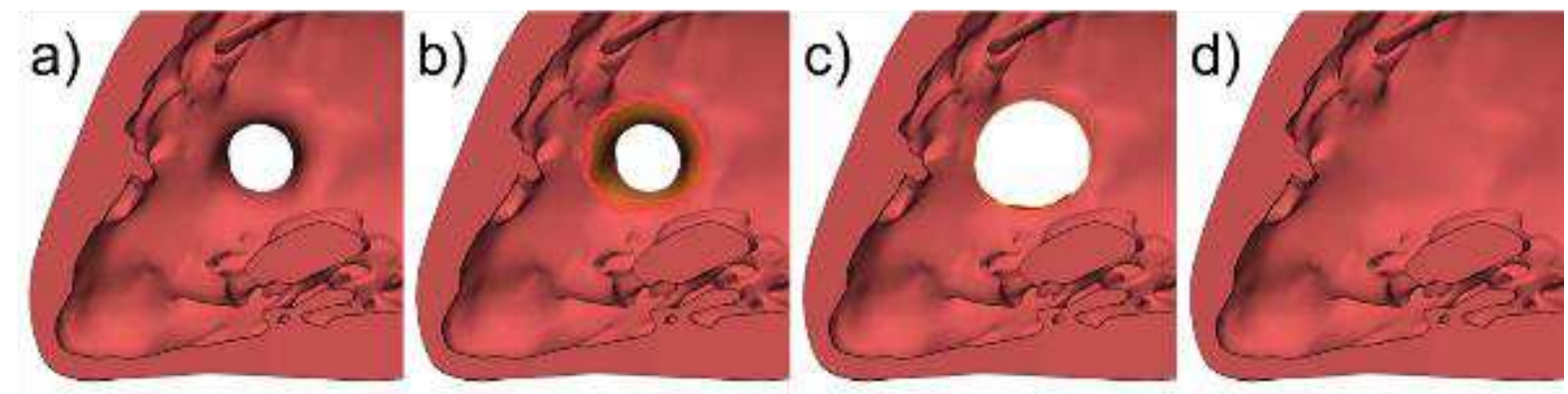

FIGURE 5: a) Holes or connections in 3D anatomical models can be closed virtually, in this case a VSD. b) Firstly, the surface around the hole is marked. c) The marked area is then deleted resulting in an enlargement of the primary defect. d) Occlusion of the defect and post-processing of the edited area masks the transition of old to new surfaces

Another design functionality relevant for the creation of anatomical variations and concurrently for 3D printing per se is the local adaptation of wall thickness and structure size. Locally changing the wall thickness is particularly relevant for hollowed models or the atrial or ventricular septum. The local manipulation of size can be applied to single structures such as the atria, ventricles and vessels instead of the complete heart. Furthermore, if the 3D model is created with the intention of being printed in $3 D$, specific design rules must be taken into account. The 3D printing technique and material used determine the resolution and minimal possible wall thickness required. Thus, structures which are thinner than the required minimum printing thickness must be virtually dilated to avoid printing failure or model breakage. Creating a local change 
in thickness can be acquired by marking a selected area and applying local offset operations. Post-processing by smoothing the surface and improving the triangle mesh of the STL makes the transition between the retained and the modified surface invisible. The local creation of an offset can be utilized to emphasize the valvular structures such as the leaflets of the aortic or pulmonary valve or the rim of the mitral or tricuspid valve by applying the offset along a line or curve instead of a marked area. The workflow of dilating the coronaries of a heart model by applying a local offset to a defined area is shown in figure 6.

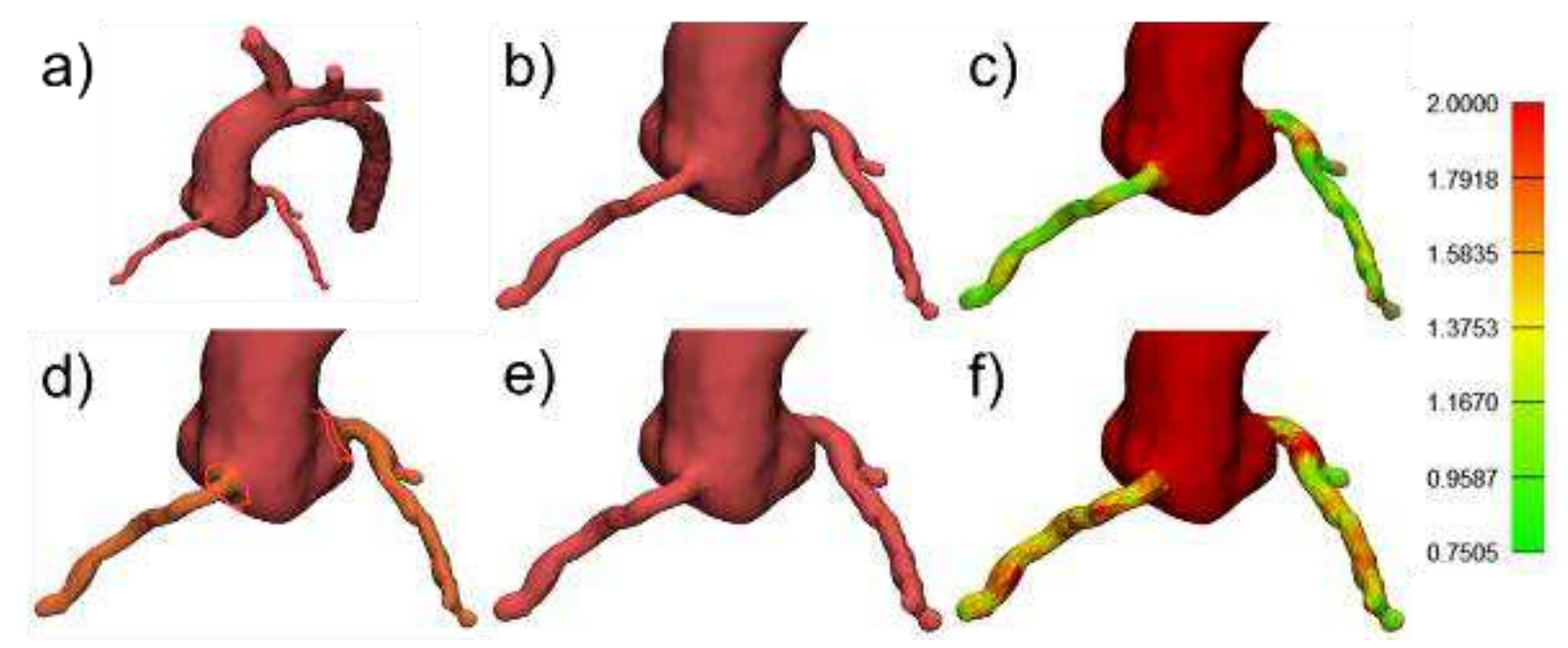

FIGURE 6: a) The images show a 3D model of the aorta. b) Delicate structures, in this case the coronaries, are often too thin for 3D printing. c) Analytical measurements can help to assess the wall thickness of a $3 D$ model. The coronaries of this model commonly have a wall thickness $<1 \mathrm{~mm}$ (according to the color scale). $d$ ) The area that needs to be dilated is marked on the surface of the STL. e) Then, by applying a local offset operation, the marked triangles are shifted towards the outside. The transition area is post-processed to conceal the surface modification. f) Renewed analysis shows that the coronary thickness is $>1 \mathrm{~mm}$, the critical value for most $3 D$ printing technologies. 
The wall thickness of thin vessels can by dilated, however, for tubular structures such as long or peripheral vessels extending in length or changing their pathway can also be of relevance, for example, in 3D training models for catheterization. Anatomical modification requires definition of the adapted (longer and/or different) pathway of the vessel as well as of the outer diameter (globally or locally). The resulting tubular structure has a smooth cylindrical shape which appears more realistic by including irregularities into the surface through local adaptation. An example of creating a tubular vessel structure and giving it a realistic appearance is shown in figure 7.
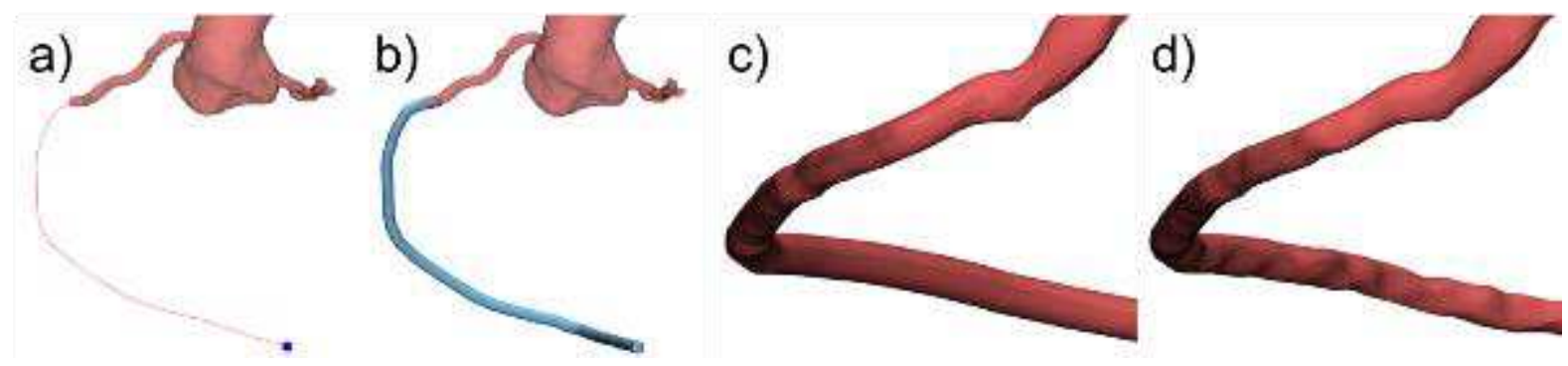

FIGURE 7: a) For the creation of a vessel, e.g. a coronary, its pathway and diameter need to be defined. b) Based on the resulting geometrical information a tubular structure is created. c) The automatically generated surface has a smooth, cylindrical shape letting it appear unnaturally. d) However, post-processing allows to make the surface structure of the computed tube more anatomical and realistic.

Another example of a helpful tool to perform engineering on anatomy are Boolean operations. These are required to create a heart model representing not only a hollowed version of the blood volume but also including the heart muscle. A 3D heart model is originally based on the segmentation of the blood volume visible in a CT or MRI dataset. Hence, the hollow model of the heart does not automatically represent the muscular structures of the myocardium and intraventricular septum. The hollowing is performed by adding an artificial wall of uniform thickness to the outside of the blood volume. For vessel structures there is no other way to achieve a vascular wall due to 
the restrictions given by the resolution of the 2D images. In general, the pixel size and slice thickness for angiographic CT scans of congenital heart disease lies at between $0.5 \mathrm{~mm}$ and $3 \mathrm{~mm}(28)$, whereas the slice thickness in MRI imaging lies at between 3 $\mathrm{mm}$ and $5 \mathrm{~mm}$ (29). The wall thickness of the aortic vessel, commonly the strongest and thickest vessel in the human body, ranges between $1.5 \mathrm{~mm}$ and $2 \mathrm{~mm}$ in adults. (30) In children these parameters are below or at the lower limit of the reference ranges determined for adults, e.g. the wall thickness of the pulmonary artery (PA) assessed by optical coherence tomography (OCT) imaging is $0.19 \pm 0.06 \mathrm{~mm}$ for pediatric patients at the age of $2.0 \pm 2.0$. (31) Therefore, the real vessel wall is too thin to be clearly represented in clinical CT and MRI scans due to the loss of information in between image slices. Thus, the vascular wall often cannot be segmented but must be virtually engineered. For the myocardium conditions are different as it is visible in 2D scans enabling the segmentation and modeling of the original, physiological anatomy of the heart muscle. However, this process is not trivial and requires extensive postprocessing, to achieve a smooth muscular surface which at the same time denatures the intraventricular surface. Consequently, the STL of the myocardium and the STL of the hollow blood volume are combined in a final step. A series of Boolean operations allows achieving a correct representation of the heart muscle and the endocardial surface in one 3D heart model. Parts of this complex workflow are illustrated in figure 8. 

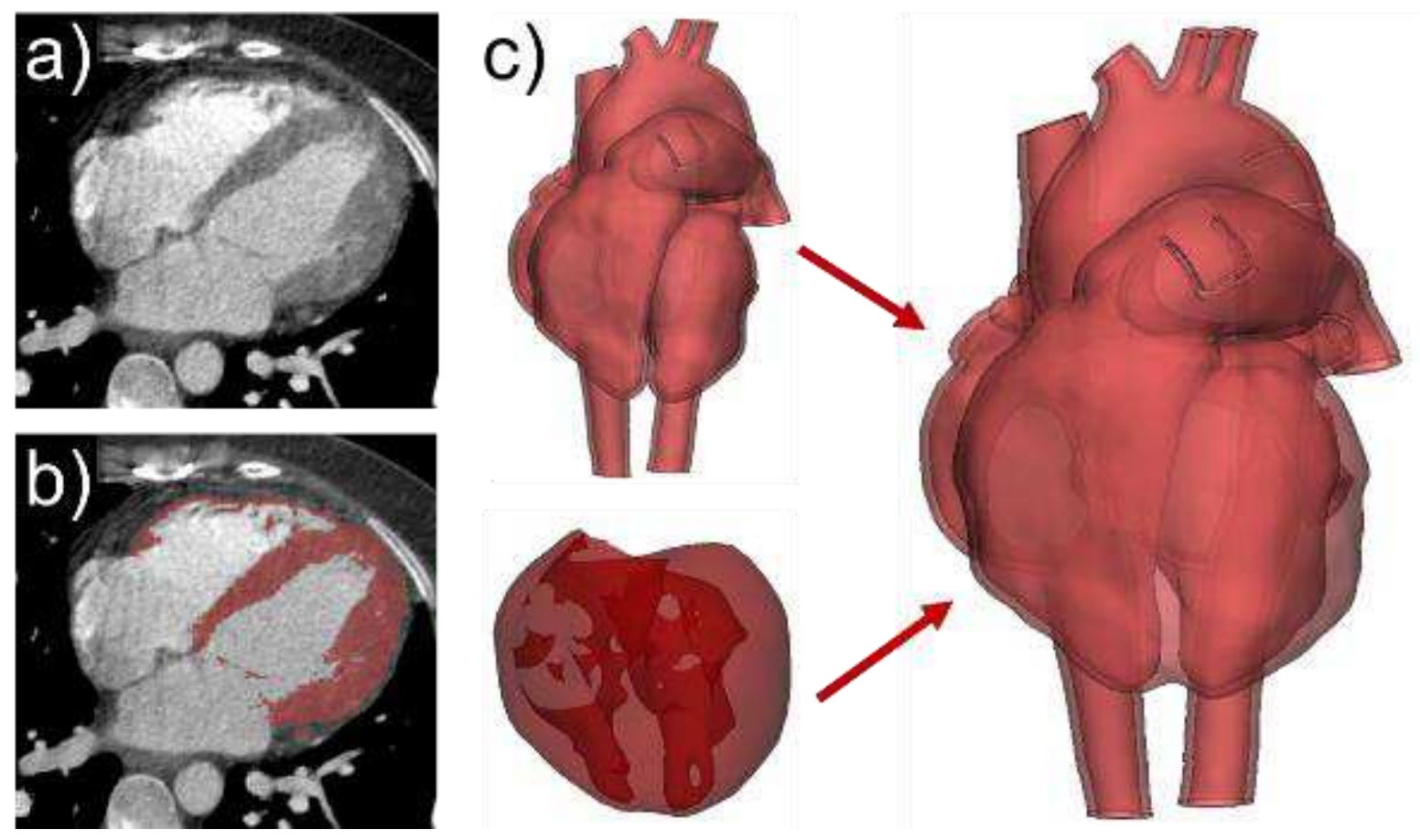

FIGURE 8: a) In contrary to the vascular walls the myocardium is visible in CT images.

b) Segmentation of the heart muscle is performed by local thresholding techniques. c) Extensive post-processing is required to achieve a smooth outer surface of the myocardium. To maintain the correct anatomical structure of the ventricles in the final model the hollow blood volume and the smoothed myocardium are then combined by a series of Boolean operations.

\section{Results}

The presented techniques for the creation of various 3D heart models has been utilized for catheter training courses in pediatric cardiology. In the following, four examples of engineered CHD models are presented:

1) Heart model with a PDA augmented by perimembranous and muscular VSD (pmVSD, mVSD)

2) Right heart model with pulmonary valve stenosis (PS) augmented by the left heart and myocardium 
3) Series of heart models with ASD showing the hemodynamic effect on the right atrial and ventricular wall

4) Left heart model with isthmus stenosis augmented with an engineered aortic valve

1) Heart model with a PDA augmented by pmVSD and mVSD: To safe printing time and material a 3D heart model originating from a dataset with PDA was augmented by two septal defects between the ventricles, a pmVSD and $\mathrm{mVSD}$ respectively. The 3D heart was printed for teaching purposes as an illustrative model for the demonstration of the morphology of the PDA and VSD. To mimic the correct anatomical appearance of a ventricle septum defect, a cone shaped hole with larger opening towards the left ventricle was created. Thereby, the hemodynamic influence of the septal defect resulting from higher pressure on the left heart was taken into account. Instead of using a geometrical, symmetric cone to design a simple hole, circular curves were positioned on each side of the septum representing the inlet and outlet of the VSD. By connecting these curves with a tubular surface, a negative of the septal defect was generated. The 3D object was then subtracted from the original heart model by Boolean operations. Finally, the edges of the resulting hole were smoothed to obtain a more natural and realistic shape. The procedure was equally performed for the pmVSD and the mVSD respectively.

With regards to the teaching aspect of the model windows were included in the 3D heart. As the model was intended for 3D printing in a flexible material, flap-shaped windows were designed, which allowed opening for insight but at the same time a complete representation of the outer heart surface when closed. The contours of the flap-shaped windows were first defined and drawn onto the surface of the heart model. A 3D object representing the outline of the window-flap was created based on the afore 
added contours and subtracted from the original model. This process was repeated for every window in the teaching model. The engineering process for the PDA heart model with two VSDs is depicted in figure 9.
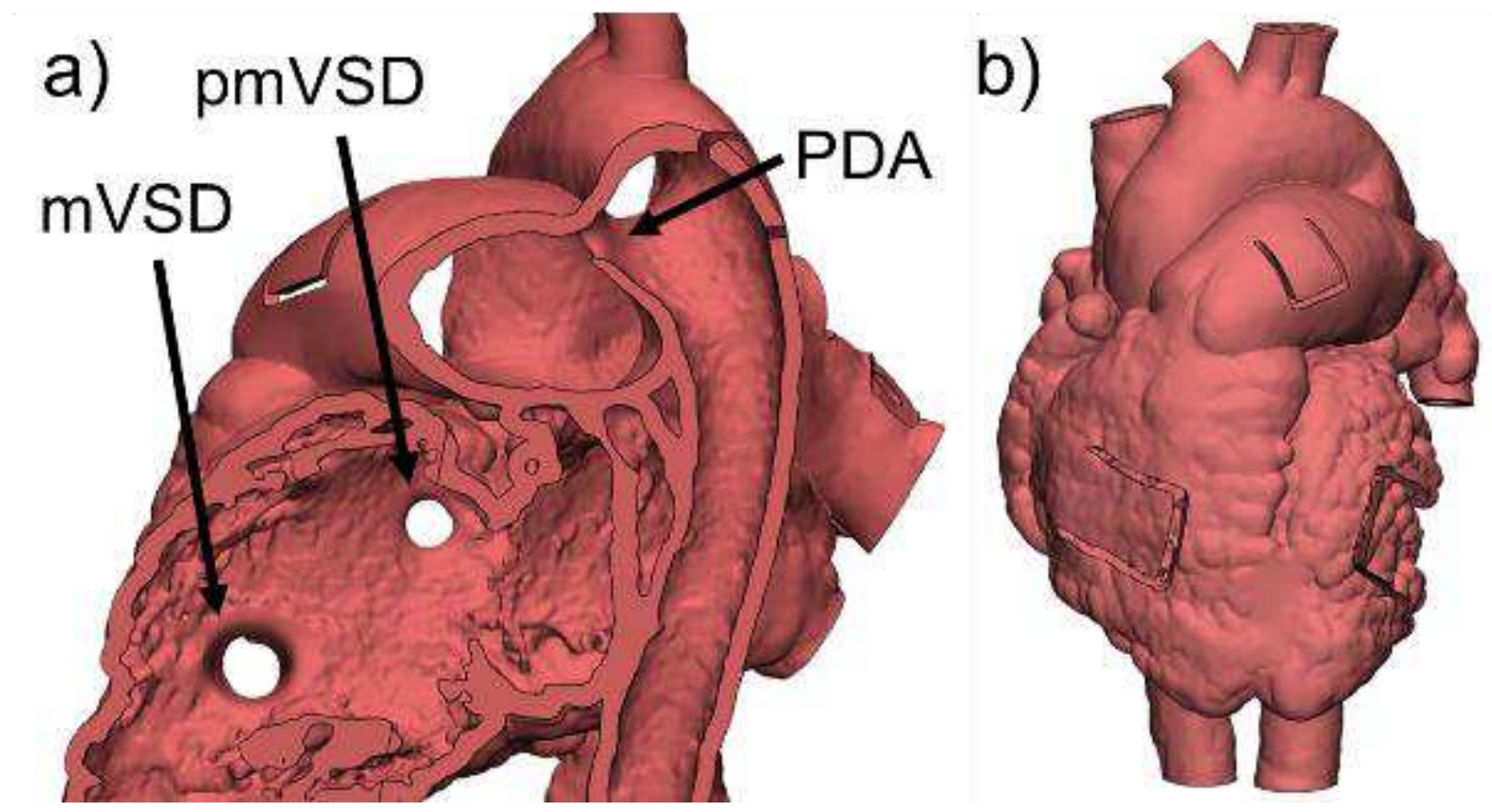

FIGURE 9: a) For teaching purposes a 3D heart model from a patient with PDA was augmented with two ventricle septum defects, a pmVSD and mVSD respectively. b) Additionally, flap-shaped windows were included into the design to provide insight into the model once $3 D$ printed in a flexible material.

2) Right heart model with PS augmented by the left heart and myocardium: The design of the heart model described in this section was changed and optimized in several iterations. As the 3D heart was designed for and utilized in hands-on catheter training courses for students, residents and pediatric cardiologists the changes were mostly based on feedback gathered from the participants of these courses. Additional modification was performed to adapt the complexity of the 3D training models to the skill level of the trainees as well as the learning aims of each course. The original dataset represented a heart with a kink in the left PA. From this dataset a 3D model of 
the right heart with the right atrium and ventricle, inferior and superior vena cava (IVC, SVC) as well as the pulmonary left and right artery (without kink) was created.

Subsequently a first 3D catheter training model with PS for practice of balloon dilatation was designed. The pulmonary valve was given a bulky shape to mimic the morphological transformation caused by the stenosis over time. Additionally, constricting walls with central holes were added at the positions of the tricuspid and pulmonary valve. The aim of this simple representation of a valve was to create a barrier for the catheter when moving forward from one heart structure to another simulating the haptic impression of passing a valve in catheterization on a real patient's heart. Finally, the intraventricular structures were smoothed extensively to make catheter manipulation inside the heart model easier and to avoid the tip of the catheter getting stuck in trabecular structures.

The second version of this training model was created for a Klinikpartnerschaften (clinical partnerships) project funded by the GIZ (Deutsche Gesellschaft für Internationale Zusammenarbeit $\mathrm{GmbH}$ ). Within the scope of the two-year project together with the Ayder Referral University Hospital in Mekelle, Ethiopia, three pediatric physicians with no experience in cardiology or cardiac catheter training were trained in the basics of diagnostic and interventional catheterization of patients suffering from congenital heart disease. The aim is to transfer the required knowledge and skills to such an extent that the Ethiopian pediatric cardiologists are able to manage the most common cases of congenital heart disease in the future by treating their patients independently in their cath lab. During the learning process, the team starts training the procedure for each $\mathrm{CHD}$ case on the respective 3D heart model. The simulation training is continued until the first and second operator are both familiar with their tasks and able to correctly follow the procedure and handle the devices. The acquired skills 
are then directly transferred to patient interventions under supervision of the German trainers. For continuous training the 3D heart models remain in the hospital in Ethiopia and the team regularly reports about their training progress. After the two-year project the pediatric cardiologists should be qualified to treat their patients independently. The first procedure trained was the interventional treatment of PS via balloon dilatation. The right heart model with PS was therefor augmented with the left heart structures as well as the myocardium, both derived from the original CT scan, to create an entire and more realistic representation of the heart. The heart model with pulmonary valve stenosis was provided in three sizes representing an adult, adolescent and infant patient. Figure 10 shows two of the Ethiopian doctors practicing catheterization on the 3D printed infant heart model.

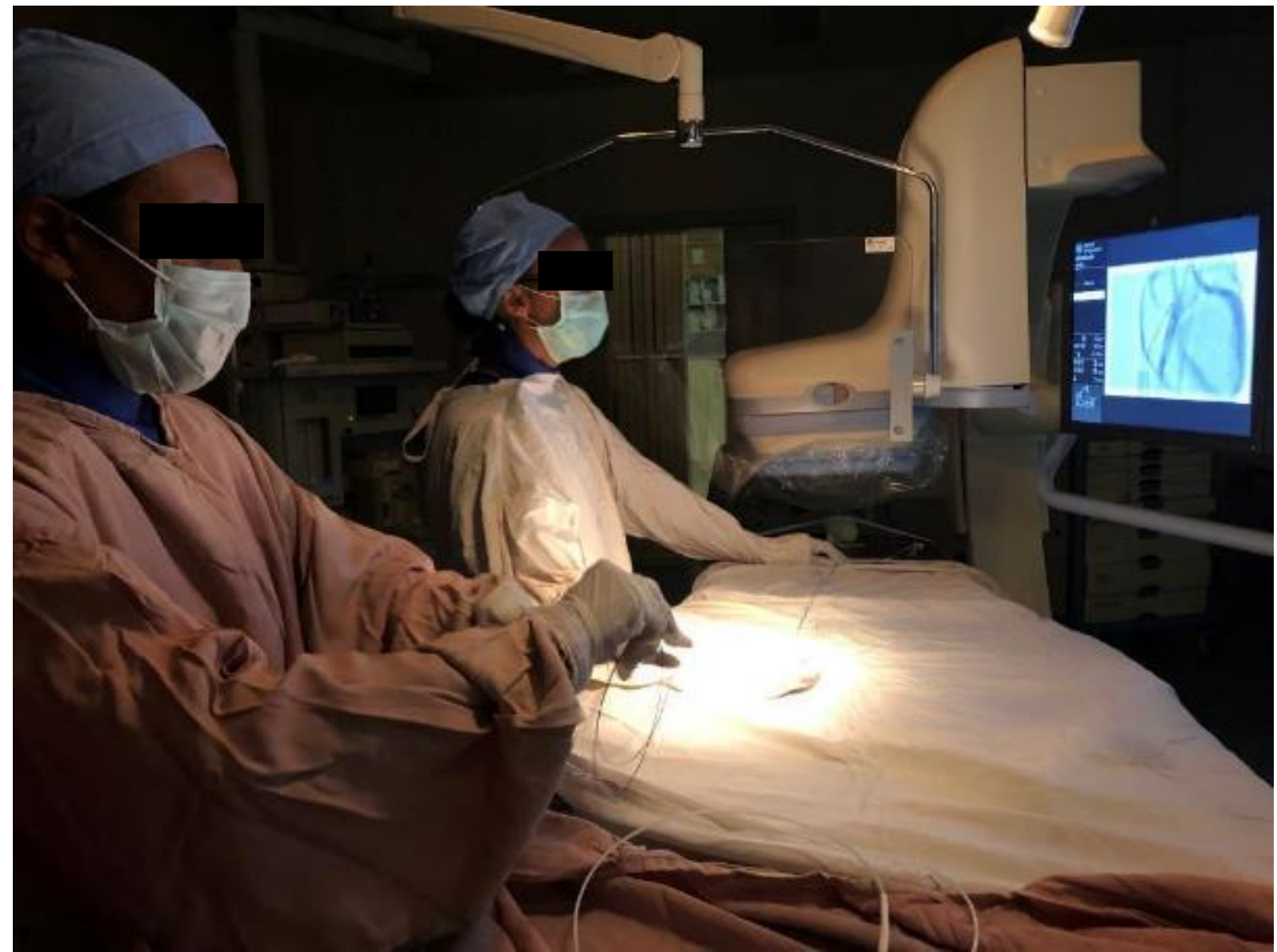


FIGURE 10: Within the scope of a partnership project with the Ayder Referral University Hospital Mekelle, Ethiopia, pediatric physicians are trained in diagnostic and interventional pediatric cardiology. The training is performed on $3 D$ printed heart models representing different cases of CHD. The models allow to practice the manipulation of the catheter and the tasks of first and second operator during the procedure before the acquired skills are transferred to real patients. The image shows two pediatric cardiologists training on an infant 3D printed heart model with PS.

Based on the experiences gathered during the training in Ethiopia and feedback from workshops in Europe the 3D model with PS was iteratively optimized by further design modifications. Primarily, the right ventricular outflow track (RVOT) was adapted to a more funnel-shaped structure. At the same time the pulmonary valve was improved by replacing the plane wall with a hole by a more representative valvular structure. These design adaptations allowed a guided entrance from the right ventricle into the PA with the catheter and presented more realistically in X-ray imaging. Finally, the catheter pathway from the IVC to the right atrium and subsequently through the tricuspid valve to the right ventricle was optimized. Based on the feedback of proficient pediatric cardiologists the right heart structures were modified such that a more physiological catheter trajectory was created. Figure 11 shows a contour overlay of the designed PA (stenosis, thickness adjusted, no kink) of the 3D PS model onto an axial slice of the original CT images. Moreover, the first and latest version of the 3D heart model for catheter training are pictured. 


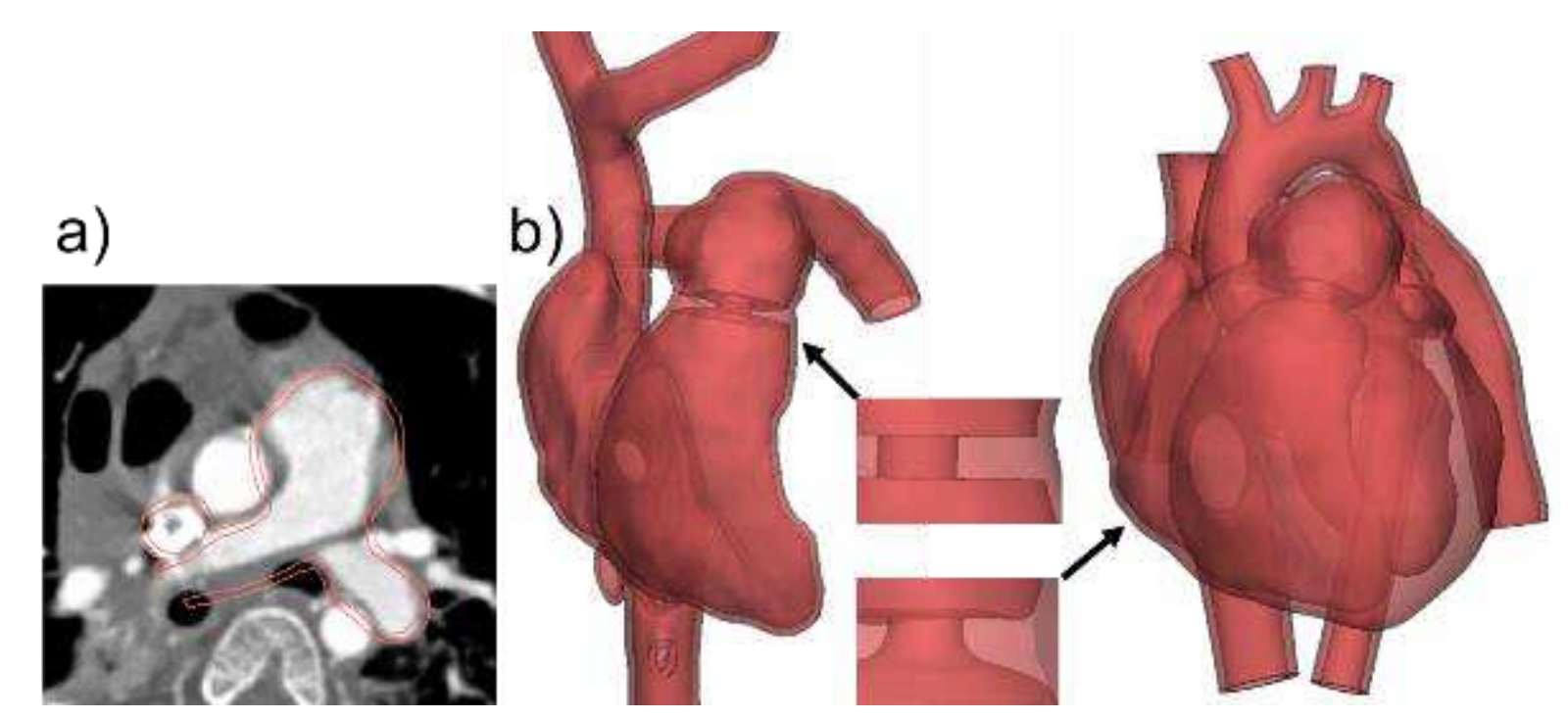

FIGURE 11: a) Computer aided design tools allow changing the anatomical structure of 3D models. The image shows the contours of the adapted and hollowed 3D heart model projected onto the axial image slice of the CT scan it was segmented from. b) The catheter training model was iteratively modified. The first version represented only the right heart with simplified PA. For the latest version the left heart structures and myocardium were added, the pathway towards the pulmonary valve was improved for smooth and realistic catheter manipulation and the shape of the valve was made more realistic.

3) Series of heart models with ASD showing the hemodynamic effect on the right atrial and ventricular wall: Teaching models are helpful in illustrating the effects of intracardial pathologies on the cardiac tissue over time by representing various progressive stages in the 3D models. As an example, 3D heart models illustrating the possible hemodynamic impact of an atrial septum defect to the right atrium and ventricle have been designed. The malfunction of the septum, the barrier between the left and right heart, in combination with the pressure gradient results in a left-to-right shunt across the ASD. Therefore, the blood volume in the right heart increases and must be pumped in addition to the normal volume. However, the right heart structures cannot withstand the additional mechanical load. In the end, the right atrium and 
ventricle will dilate over time due to the defect in the atrial septum. Without the use of engineering CAD software, invasive imaging of one patient at different stages over time or of different patients with the same pathology but at different stages in the process of disease would be required to illustrate the progression of the disease caused by the ASD by 3D printed heart models. To avoid unnecessary image acquisition and extensive time-consuming database search the heart structures were adapted virtually. The dataset used for the models was again the CT scan of the patient with PDA described in the first example, thus, with an intact atrial septum. The PDA between aorta and PA was closed for the ASD model. In exchange, a hole in the atrial septum was created. The first ASD model represented a heart not suffering from the hemodynamic effects of the ASD for a long time. For the second model the right atrium and ventricle were dilated to show the beginning enlargement due to increased mechanical load. Further dilation of the right heart structures was performed to design a third stage of the ASD heart. The transition between the retained and the adapted surface of the 3D model was improved by post-processing such as smoothing and rearrangement of the STL triangles. The three heart models representing the stages of the right heart changing its shape due to the hemodynamic effects of an ASD are pictured in figure 12 .
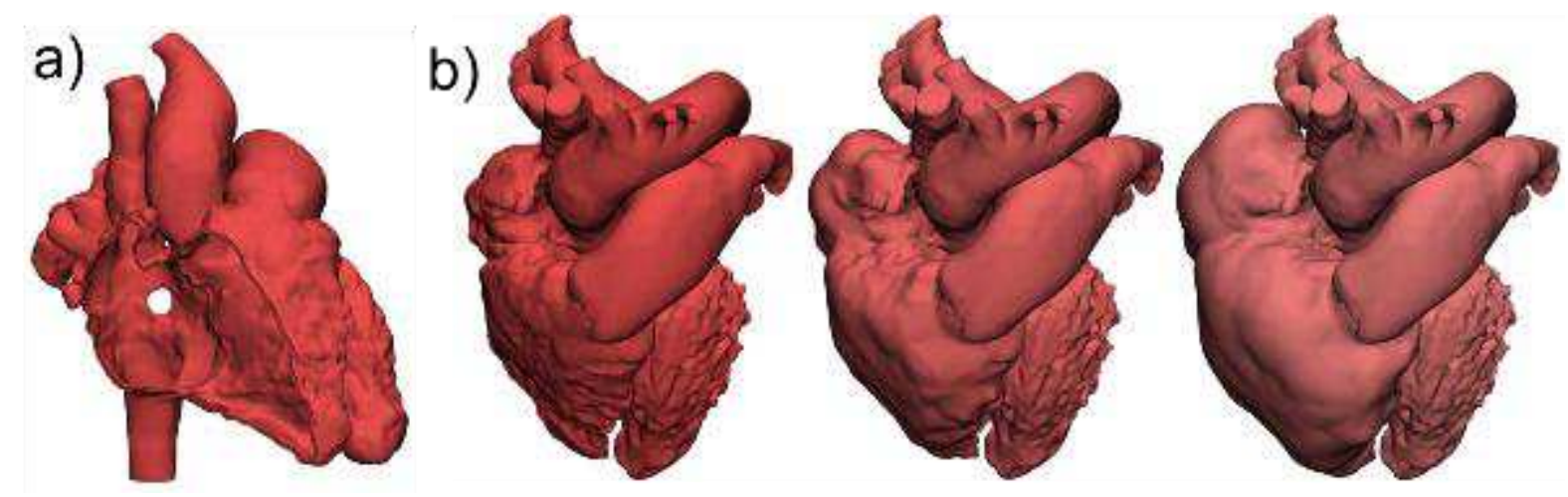

FIGURE 12: a) A 3D heart model with ASD was engineered based on the dataset of a patient with PDA. b) The hemodynamic effect of an ASD leads to progressive dilation 
of the right atrium and ventricle due to increased blood volume migrating from the highpressure left heart to the right heart side. This has been illustrated by $3 D$ heart models representing different stages of the disease.

4) Left heart model with isthmus stenosis augmented with an engineered aortic valve: The last example presented in this paper is a 3D heart model designed for hands-on advanced catheter training courses on a beating heart model. A left heart model with stenosis at the aortic isthmus was therefor designed to be connected to a closed circulation system filled with water and stimulated with pulsatile flow. The pulsatility is induced by an artificial ventricular assist device (VAD, Berlin Heart GmbH) simulating the patient's heartbeat in the flexible 3D printed heart model allowing further training applications on 3D printed heart models for pediatric cardiology such as intracardiac pressure measurements and angiographic imaging using a contrast agent. The dataset of the 3D heart originates from the same patient with PS described in section 2). However, for the pulsatile heart only the structures of the left heart were represented in the 3D model. Additionally, the isthmus stenosis was virtually designed. After creating the hollow 3D heart model, the wall at the isthmus stenosis was enforced for resistance and durability due to intended repetition of practicing balloon dilatation. The intracardiac surface was smoothed extensively to avoid obstacles for catheter manipulation during training. In order to keep the water inside the pulsatile system the endings of the heart vessels were closed off. To maintain all vessel endings as possible connection points and for them to be compatible with the connectors of the flow system this was done by designing tubes of equal geometric measurements to the vessel endings which could be cut open, if necessary. The descending aorta and the inferior right pulmonary vein equally designed as geometric tubes remained open for direct connection to the tube connectors of the pulsatile system. The first version of this 
model was equipped with a simplified aortic valve, thus, a wall with a hole as described for the initial version of the model with PS. The design was improved by replacing the valve with an engineered STL of an aortic valve. According to the feedback of proficient pediatric cardiologists the new model design including a highly improved representation of the aortic valve provides realistic simulation conditions for training of angiographic imaging in particular. Figure 13 shows the first and latest versions of the pulsatile 3D heart model for hands-on practice of the catheterization procedure indicated for patients suffering from isthmus stenosis. Moreover, it is demonstrated how the model with a realistic aortic valve is represented under angiographic imaging.

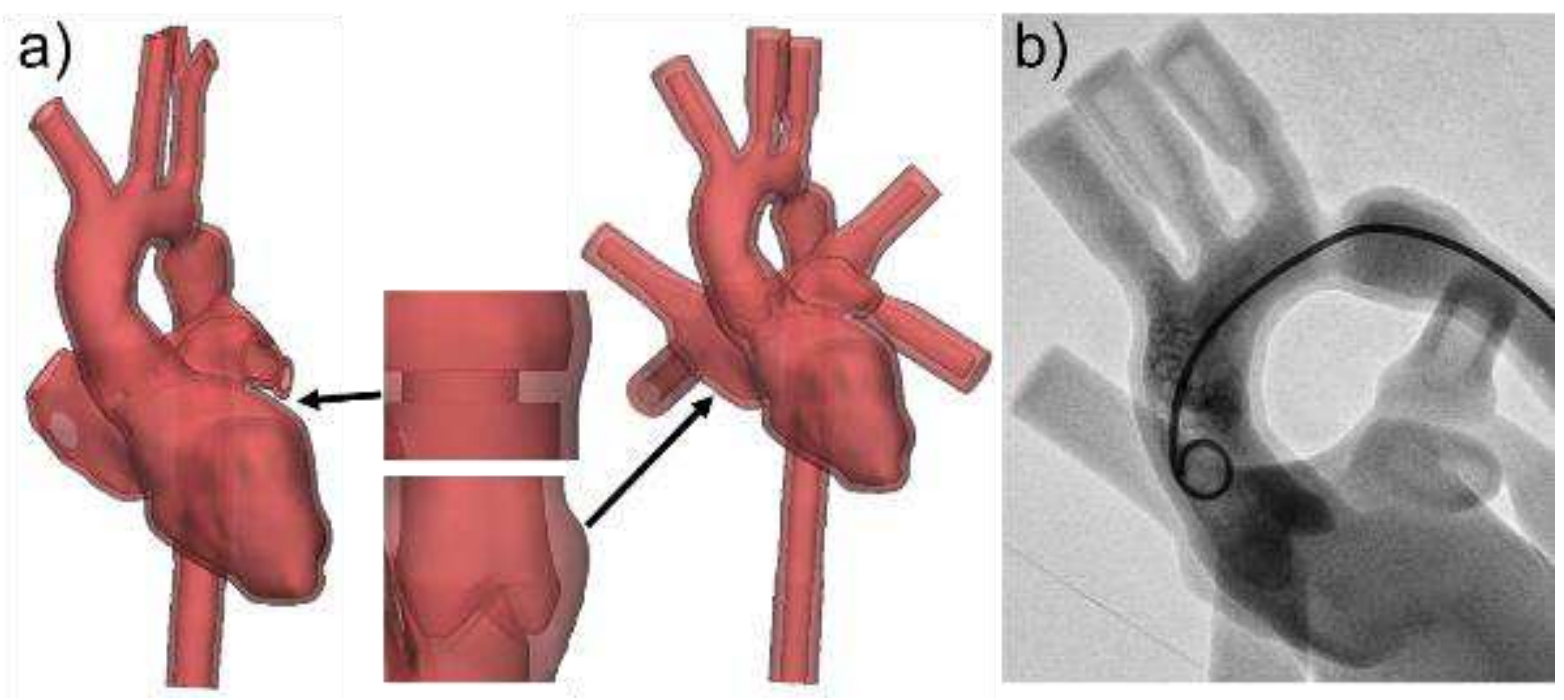

FIGURE 13: a) The design of a left heart catheter training model with engineered isthmus stenosis and simplified representation of the aortic valve was adapted for connection to a pulsatile flow system. Since the system is filled with water the vessel endings were closed off except for the descending aorta and one pulmonary vein which serve as points of connection to the system. Moreover, a realistic representation of the aortic valve was achieved by replacing the prior design by an engineered STL. b) The water filled beating heart allows advanced catheter training including angiographic imaging. 


\section{Discussion}

The investigations of this study show that it is possible to virtually adapt 3D heart models and thereby creating many different anatomical variants and CHD cases based on a single scan of a patient. The main use for these non-patient-specific 3D printed models is education and training, for example in catheter training courses for students, residents and proficient pediatric cardiologists. The feedback of the participants was very good, particularly regarding the realistic visual and haptic representation of the 3D printed heart models. The possibility of practicing the complete diagnostic and interventional procedure of catheterization in CHD patients for first and second operator was highlighted as being utmost helpful.

Applications for which the unique anatomy of a patient is of relevance require patientspecific 3D modeling. This applies to all clinical cases in which 3D models are used for planning a clinical intervention or surgery, taking measurements, sizing implants or assessing the geometrical relations of anatomical structures to determine the best approach or most adequate treatment. (3) However, there are also plenty of examples of application for 3D printed models not required to be patient-specific and therefore do not have to be based on original patient data but can be engineered. Changing the real anatomy of a 3D heart model can have various advantages. One is the possibility to include several pathologies in one model to save time, material and costs of printing. Another benefit is the adaptation of the 3D model to the level of difficulty and aims of a training course or the complexity of clinical reality. Possible applications for nonpatient-specific 3D printed models are teaching morphology to students, medical personal, patients and parent, or hands-on training in diagnostic and interventional cardiology as well as cardiac surgery. It can also be useful to prepare clinical cases on 
engineered heart models, if there is no CT or MRI scan available. In this case, a logical approach is to design a 3D model based on the available patient information, e.g. derived from ultrasound imaging. So far, the $3 D$ designed heart models have been utilized in catheter trainings for pediatric interventional cardiology. Broadening the field of applications to the afore mentioned and gathering data about the effectiveness of the training and the satisfaction of the trainees systematically has already been planned for future investigations. Next will be the implementation of a training course for pediatric cardiac surgeons using non-patient-specific 3D printed heart models.

Regarding the shape and structure of a STL any changes are possible using image processing and computer aided 3D design operations. Thus, whenever designing a non-patient-specific 3D model it is crucial for the engineer not to get out of touch with reality since there is an obvious risk of creating unrealistic anatomical findings. Therefore, a close collaboration between medical engineers as well as experienced cardiologists and cardiac surgeons is indispensable for creating anatomical 3D models. The engineer has to transfer the specifications regarding structure and intended use given by the medical professionals to the 3D model design. Thereby, an iterative process of asking for feedback and adapting the design leads to a realistic representation of a human heart without the use of patient data. This is also the case for the use of additional information, e.g. achieved by ultrasound imaging, to virtually adapt the shape of a 3D heart. This information can be helpful in creating close to patient-specific heart models, but the design should ultimately be checked up upon by experienced physicians with the ability to assess the resemblance of reality.

First applications of the non-patient-specific 3D CHD models have been successfully implemented and evaluated by questionnaires. The users provided positive feedback regarding the simulation trainings. At this point it is important to evaluate the 
resemblance of reality of the engineered 3D heart models. Moreover, investigations should be conducted on whether simplifications, such as smoothing the trabecular structures of the ventricles will put a bias on the experience of the trainees and affect future actions or decisions during an intervention on a real patient. Quantitative studies and feedback from experienced cardiologists and cardiac surgeons can help to answer these questions.

Finally, it has to be mentioned that for very complex cases of congenital abnormalities of the heart it can be extremely difficult and time-consuming to engineer 3D heart models without a CT or MRI scan with similar finding. Examples of such cases are hearts with transposition of the great arteries (TGA) or Tetralogy of Fallot (ToF). However, for these complex cases there is often an indication for image acquisition via CT or MRI. If this is not the case, a patient scan with similar pathology can be used.

\section{Conclusions}

It has been shown that the design of variations of CHD heart models for 3D printing based on a single scan of a patient by virtual engineering on anatomy is feasible. This approach was successfully applied for educational and training models for diagnostic and interventional pediatric cardiology. The main benefit of designing non-patientspecific 3D heart models is to create a range of virtual patient by avoiding the necessity of $\mathrm{CT}$ or $\mathrm{MRI}$ images in medical $3 \mathrm{D}$ printing. These imaging technologies require radiation $(\mathrm{CT})$ or possibly sedation $(\mathrm{MRI})$ and are therefore harmful for the patient. Thus, with the new methodology the benefits of 3D printed heart models can be exhausted without increasing the need for invasive imaging. Thereby, a 3D heart model originating from a patient's dataset can be simplified or amplified in complexity to adapt its appearance to training levels and aims of teaching or hands-on courses. Moreover, several pathological findings can be integrated in one model to safe printing time and 
material. Thus, only one model including all pathologies needs to be 3D printed instead of several models for each pathology. The approach of creating several 3D models based on a single 2D image stack cannot only be applied to training in pediatric cardiology, but also to other fields such as pediatric cardiac surgery. Expectedly, all medical fields can benefit from simulation training using engineered non-patientspecific 3D models without the need for invasive imaging.

\section{List of abbreviations}
ALS
Advanced Life Support
ASD atrial septal defect
CAD computer aided design
CHD congenital heart disease
CT computer tomography
EPALS European Pediatric Advanced Life Support
EPILS European Pediatric Immediate Life Support
GIZ Deutsche Gesellschaft für Internationale Zusammenarbeit GmbH
ILS Immediate Life Support
IVC inferior vena cava
MRI magnetic resonance imaging
mVSD muscular ventricular septal defect
NLS Neonatal Life Support
OCT optical coherence tomography
PA pulmonary artery
PDA patent ductus arteriosus
pmVSD perimembranous ventricular septal defect
PS pulmonary valve stenosis 


$\begin{array}{ll}\text { ROI } & \text { region of interest } \\ \text { RVOT } & \text { right ventricular outflow track } \\ \text { SVC } & \text { superior vena cava } \\ \text { TGA } & \text { transposition of the great arteries } \\ \text { ToF } & \text { Tetralogy of Fallot } \\ \text { VAD } & \text { ventricular assist device } \\ \text { VSD } & \text { ventricular septal defect }\end{array}$

\section{Declarations}

- Ethics approval and consent to participate: Not applicable.

- Consent for publication: Not applicable.

- Availability of data and materials: The datasets generated during the current study are not publicly available but are available from the corresponding author on reasonable request.

- Competing interests: The authors declare that they have no competing interests.

- Funding: Sponsorship of all author fees associated with publishing by Materialise NV.

- Authors' contributions: $\mathrm{CH}$ has developed and established different techniques to digitally design variant 3D models based on one single dataset and applied the method in various medical 3D printing projects in her working group. $\mathrm{NH}$ has made substantial contributions to the conception of the work and revised it. AJ and ATe tested and gave feedback for optimization of the design of the non-patient-specific 3D heart models. BB and ATh acquired and summarized data from hands-on catheterization courses involving the 3D models. MG 
supported in technical aspects related to 3D printing. NT supported in drafting the manuscript. All authors read and approved the final manuscript

- Acknowledgements: Not applicable.

- Authors' information: $\mathrm{CH}$ medical engineer, AJ pediatric cardiologist, AT pediatric cardiologist, MG mechanical engineer, NT cardiac surgeon, BB medical student, ATh medical student, $\mathrm{NH}$ chief physician of the Department for Pediatric Cardiology and Intensive Care

\section{References}

1. Ziv A, Root Wolpe P, Small SD, Glick S. Simulation-Based Medical Education: An Ethical Imperative. Academic Medicine 2003; 78(8):783-8.

2. Greif R, Lockey AS, Conaghan P, Lippert A, Vries W de, Monsieurs KG. Ausbildung und Implementierung der Reanimation: Kapitel 10 der Leitlinien zur Reanimation 2015 des European Resuscitation Council. Notfall + Rettungsmedizin 2015; 18(8):1016-34.

3. Anwar S, Singh GK, Miller J, Sharma M, Manning P, Billadello JJ et al. 3D Printing is a Transformative Technology in Congenital Heart Disease. Journal of the American College of Cardiology JACC: Basic Translational Science 2018; 3(2):294-312. 4. St. Pierre M, Breuer G. Simulation in der Medizin: Grundlegende Konzepte Klinische Anwendung. 2nd ed. Berlin, Heidelberg: Springer Berlin Heidelberg; 2018.

5. Ejaz F, Ryan J, Henriksen M, Stomski L, Feith M, Osborn M et al. Color-coded patient-specific physical models of congenital heart disease. Rapid Prototyping Journal 2014; 20(4):336-43. 
6. Costello JP, Olivieri LJ, Su L, Krieger A, Alfares F, Thabit O et al. Incorporating Three-dimensional Printing into a Simulation-based Congenital Heart Disease and Critical Care Training Curriculum for Resident Physicians. Congenital Heart Disease 2015; 10(2):185-90.

7. Lau IWW, Liu D, Xu L, Fan Z, Sun Z. Clinical value of patient-specific threedimensional printing of congenital heart disease: Quantitative and qualitative assessments. PLoS One 2018; 13(3):1-15.

8. Loke YH, Harahsheh AS, Krieger A, Olivieri LJ. Usage of 3D models of tetralogy of Fallot for medical education: impact on learning congenital heart disease. BMC Medical Education 2017; 17(54):1-8.

9. Olivieri LJ, Su L, Hynes CF, Krieger A, Alfares FA, Ramakrishnan K et al. "Just-InTime" Simulation Training Using 3-D Printed Cardiac Models After Congenital Cardiac Surgery. World J Pediatr Congenit Heart Surg 2016; 7(2):164-8.

10. Su W, Xiao Y, He S, Huang P, Deng X. Three-dimensional printing models in congenital heart disease education for medical students: a controlled comparative study. BMC Med Educ 2018; 18(1):1-6.

11. Wang Z, Liu Y, Luo H, Gao C, Zhang J, Dai Y. Is a Three-Dimensional Printing Model Better Than a Traditional Cardiac Model for Medical Education? A Pilot Randomized Controlled Study. Acta Cardiol Sin 2017; 33(6):664-9.

12. White SC, Sedler J, Jones TW, Seckeler M. Utility of three-dimensional models in resident education on simple and complex intracardiac congenital heart defects. Congenit Heart Dis 2018; 13(6):1045-9. 
13. Biglino G, Capelli C, Koniordou D, Di Robertshaw, Leaver L-K, Schievano S et al. Use of 3D models of congenital heart disease as an education tool for cardiac nurses. Congenit Heart Dis 2017; 12(1):113-8.

14. Costello JP, Olivieri LJ, Krieger A, Thabit O, Marshall MB, Yoo SJ et al. Utilizing Three-Dimensional Printing Technology to Assess the Feasibility of High-Fidelity Synthetic Ventricular Septal Defect Models for Simulation in Medical Education. World J Pediatr Congenit Heart Surg 2014; 5(3):421-6.

15. Beckerman Z, Mery CM. Teaching congenital heart disease: A new era? J Thorac Cardiovasc Surg 2017; 153(6):1541.

16. Yoo SJ, Spray T, Austin, E. H., 3rd, Yun TJ, van Arsdell GS. Hands-on surgical training of congenital heart surgery using 3-dimensional print models. J Thorac Cardiovasc Surg 2017; 153(6):1530-40.

17. Yoo SJ, Thabit O, Kim EK, Ide H, Yim D, Dragulescu A et al. 3D printing in medicine of congenital heart diseases. 3D Print Med 2015; 2(1):1-12.

18. Stanbridge RdL, O'Regan D, Cherian A, Ramanan R. Use of a pulsatile beating heart model for training surgeons in beating heart surgery. Heart Surg Forum 1999; 2(4):300-4.

19. Jonas RA. Training fellows in paediatric cardiac surgery. Cardiol Young 2016; 26(8):1474-83.

20. Burkhart HM. Simulation in congenital cardiac surgical education: We have arrived. J Thorac Cardiovasc Surg 2017; 153(6):1528-9.

21. Biglino G, Capelli C, Wray J, Schievano S, Leaver LK, Khambadkone S et al. 3Dmanufactured patient-specific models of congenital heart defects for communication in clinical practice: feasibility and acceptability. BMJ Open 2015; 5(4):1-8. 
22. Biglino G, Koniordou D, Gasparini M, Capelli C, Leaver LK, Khambadkone S et al. Piloting the Use of Patient-Specific Cardiac Models as a Novel Tool to Facilitate Communication During Cinical Consultations. Pediatr Cardiol 2017; 38(4):813-8. 23. Biglino G, Capelli C, Leaver L-K, Schievano S, Taylor AM, Wray J. Involving patients, families and medical staff in the evaluation of 3D printing models of congenital heart disease. Communication \& Medicine 2016; 12(2-3):157-69.

24. Brunner B, Thierij A, Lehner A, Fernandez S, Jakob A, Leuner C et al. Clinical implementation of a model-based training course to reduce the learning curve in interventional therapy of congenital heart defects. Seville, Spain; 2019. (53 Annual Meeting of the Association for European Peadiatric and Congenital Cardiology).

25. Thierij A, Brunner B, Lehner A, Grab M, Thierfelder N, Fernandez S et al. Establishment of a 3-D silicone model to facilitate realistic hands-on-training for diagnostic and interventional pediatric cardiac catheterization. Seville, Spain; 2019. (53 Annual Meeting of the Association for European Peadiatric and Congenital Cardiology).

26. Lau I, Sun Z. Three-dimensional printing in congenital heart disease: A systematic review. J Med Radiat Sci 2018; 65(3):226-36.

27. Applegate KE, Cost NG. Image Gently: a campaign to reduce children's and adolescents' risk for cancer during adulthood. J Adolesc Health 2013; 52(5 Suppl):93-97.

28. Baert AL, Knauth M, Sartor K, Lucaya J, Strife JL. Pediatric Chest Imaging. Berlin, Heidelberg: Springer Berlin Heidelberg; 2008. 
29. Ntsinjana HN, Hughes ML, Taylor AM. The role of cardiovascular magnetic resonance in pediatric congenital heart disease. Journal of Cardiovascular Magnetic Resonance 2011; 13:51.

30. Rosero EB, Peshock RM, Khera A, Clagett P, Lo H, Timaran CH. Sex, race, and age distributions of mean aortic wall thickness in a multiethnic population-based sample. J Vasc Surg 2011; 53(4):950-7.

31. Homma Y, Hayabuchi Y, Ono A, Kagami S. Pulmonary Artery Wall Thickness Assessed by Optical Coherence Tomography Correlates With Pulmonary Hemodynamics in Children With Congenital Heart Disease. Circ J 2018; 82(9):23507. 


\section{Figures}

\section{Common Imaging Issues}

Image Processing Solutions

- poor image resolution

- inhomogeneous distribution of contrast agent

- metal artefacts

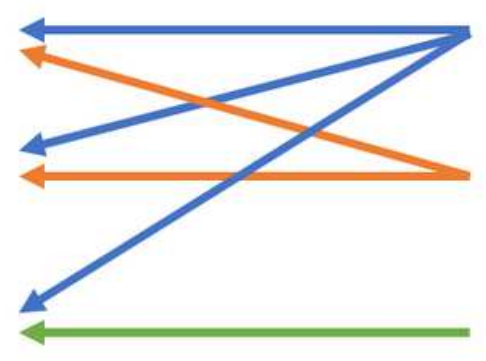

- local thresholding of a defined limited area

filling algorithms involving edge detection

Boolean operations applied to ROls with different threshold ranges

\section{Figure 1}

Various image processing solutions are provided to overcome imaging issues. Boolean operations between ROls with different threshold ranges support the segmentation of datasets with metal artefacts. Filling algorithms involving edge detection are helpful for images with poor resolution or inhomogeneously distributed contrast agent. Local thresholding is utilized for advanced segmentation in all said cases.
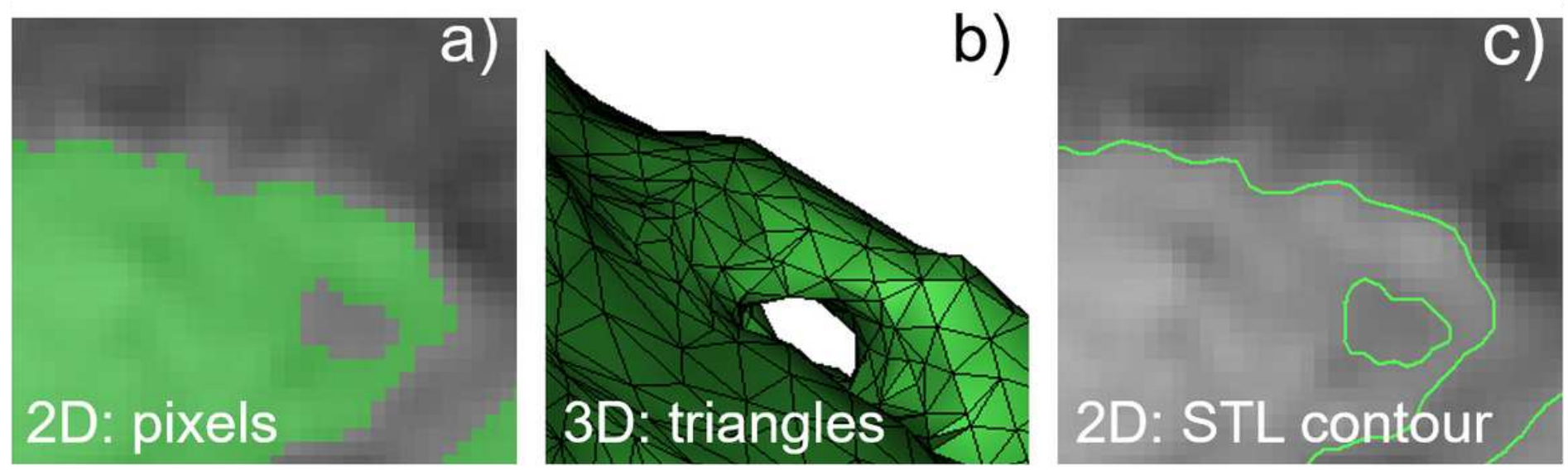

Figure 2

) Pixels marked in green on one slice of a 2D image stack. Marked pixels on subsequent slices build up voxels in 3D. b) STL surface file calculated from those voxels. The surface is defined by triangles. c) The contour of the STL can be visualized in the 2D images for control of the resemblance of reality. The calculation of the STL file is the basis for further design adaptations 

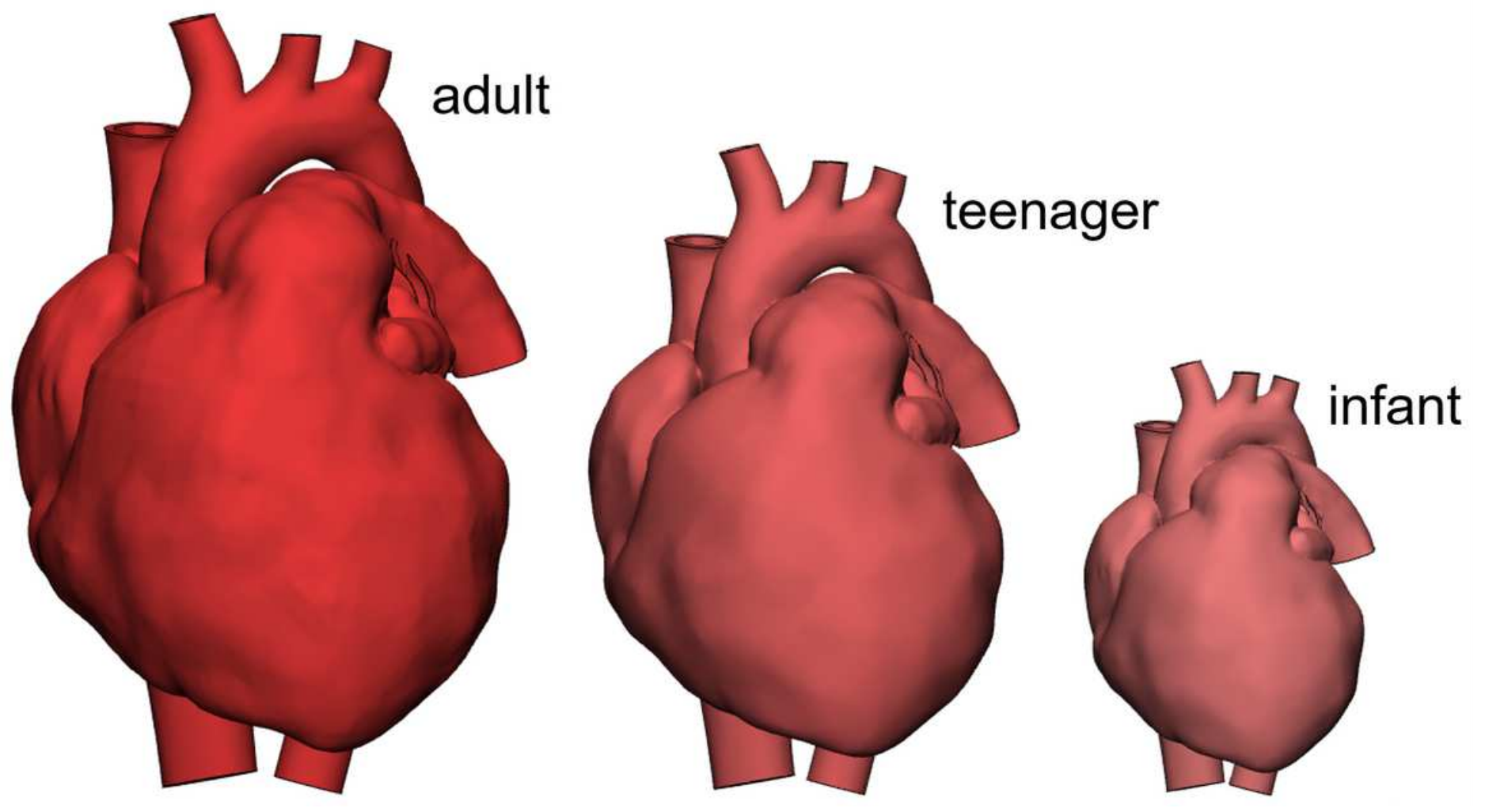

Figure 3

3D heart models of different sizes. The adult sized heart was created based on a CT scan. For the teenager and infant hearts the original model was scaled to enlarge the patient spectrum depicted in training models. Therefore, three different complexity levels for training were generated from a single scan of a patient.

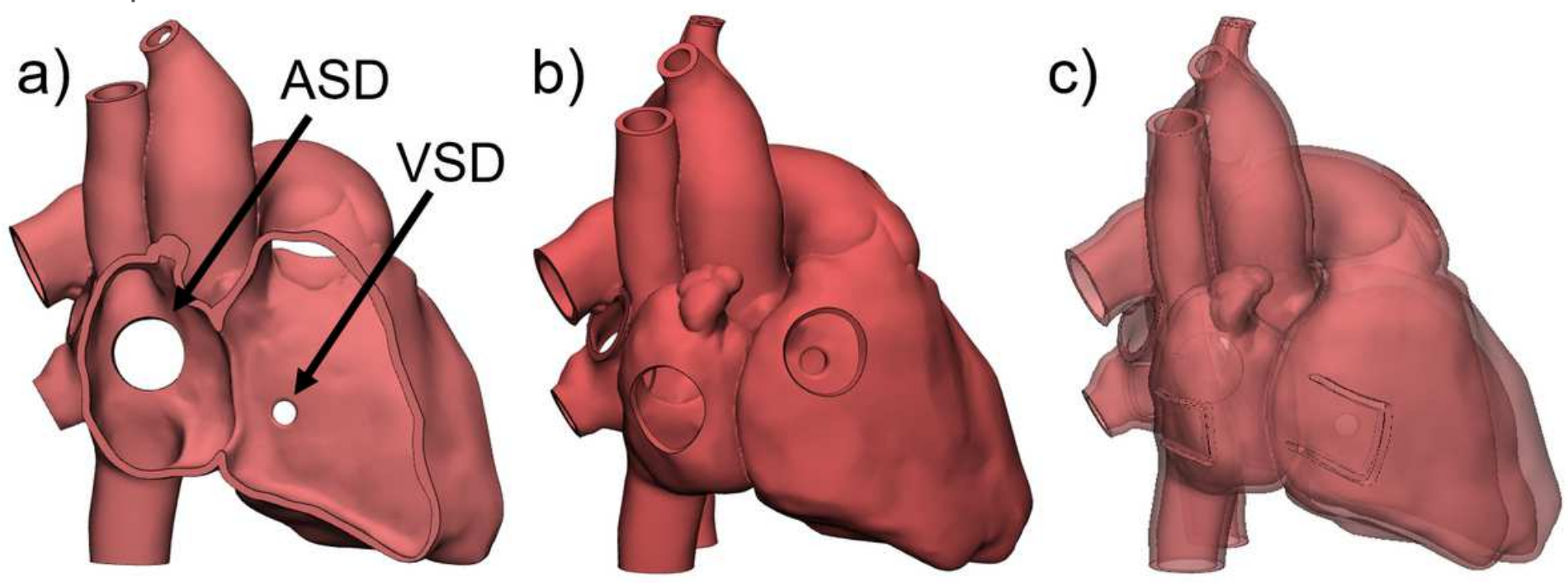

Figure 4

The vessels of all depicted 3D models have been opened by trimming operations on the respective STL. a) Cutting off larger parts and whole structures of the heart allows clear visualization of anatomical structure, in this case the septum and septal defects (atrial septal defect (ASD), ventricular septal defect 
(VSD)). The remaining parts of the ventricles can be either omitted or equipped with sockets for magnets to create an assembly model after 3D printing. b) Circular windows allow insight into intracardiac structures, but at the same time maintain a closed shape of the heart. c) Windows designed as flaps are appropriate particularly for models printed in flexible material. The appearance of heart models with flapwindows is even more closed and complete.
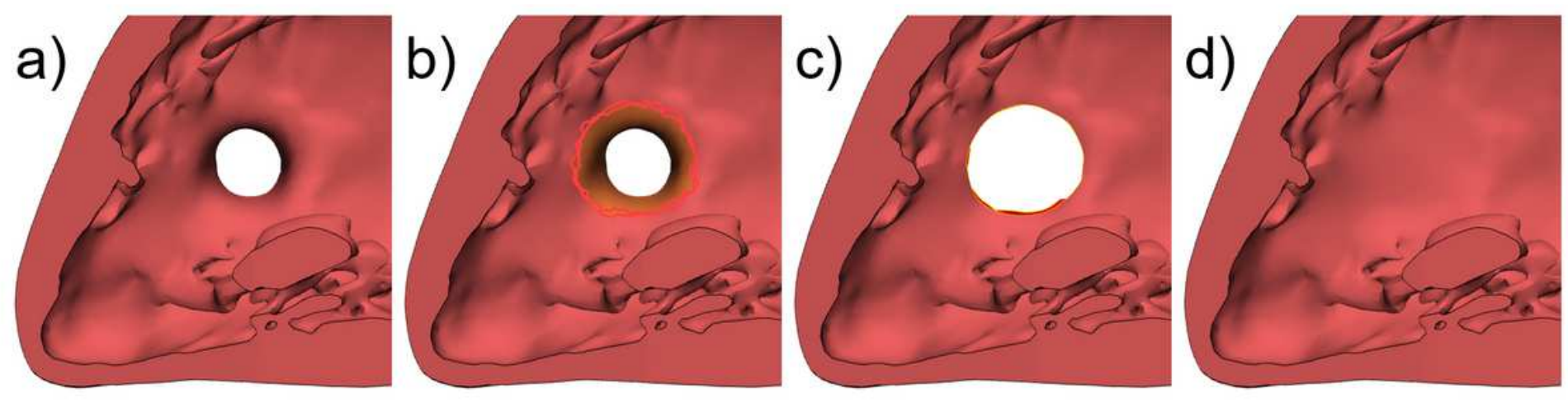

\section{Figure 5}

a) Holes or connections in 3D anatomical models can be closed virtually, in this case a VSD. b) Firstly, the surface around the hole is marked. c) The marked area is then deleted resulting in an enlargement of the primary defect. d) Occlusion of the defect and post-processing of the edited area masks the transition of old to new surfaces

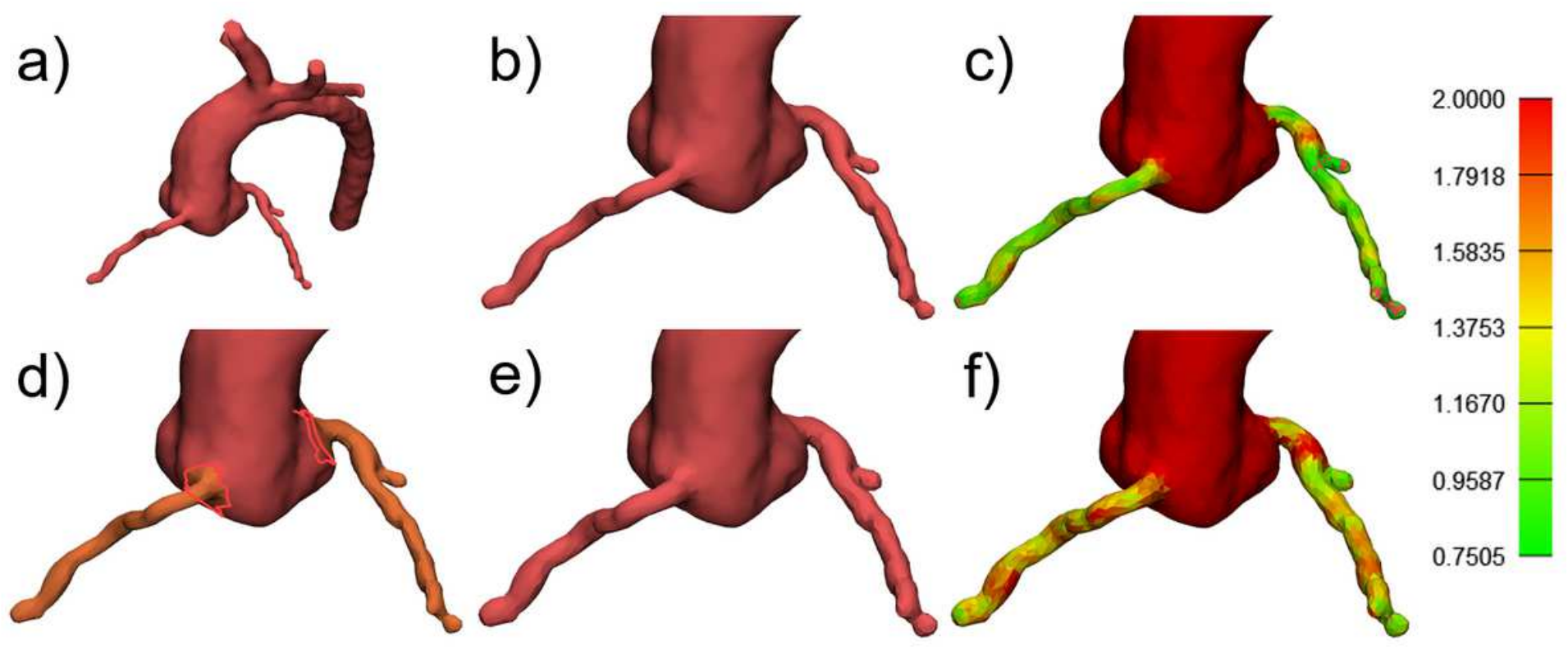

\section{Figure 6}

a) The images show a 3D model of the aorta. b) Delicate structures, in this case the coronaries, are often too thin for 3D printing. c) Analytical measurements can help to assess the wall thickness of a 3D model. The coronaries of this model commonly have a wall thickness $<1 \mathrm{~mm}$ (according to the color scale). $d$ ) The area that needs to be dilated is marked on the surface of the STL. e) Then, by applying a local offset operation, the marked triangles are shifted towards the outside. The transition area is post-processed to 
conceal the surface modification. f) Renewed analysis shows that the coronary thickness is $>1 \mathrm{~mm}$, the critical value for most 3D printing technologies.
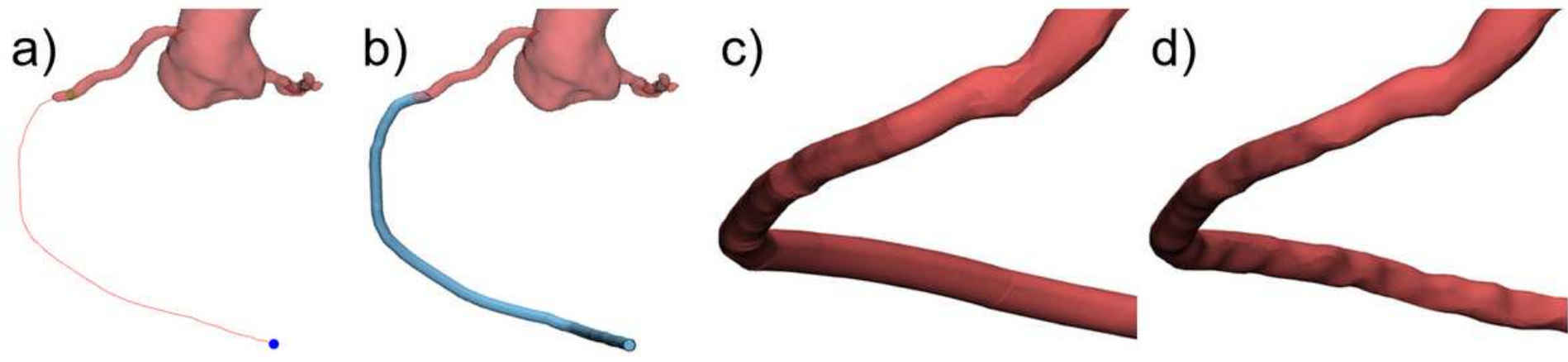

Figure 7

a) For the creation of a vessel, e.g. a coronary, its pathway and diameter need to be defined. b) Based on the resulting geometrical information a tubular structure is created. c) The automatically generated surface has a smooth, cylindrical shape letting it appear unnaturally. d) However, post-processing allows to make the surface structure of the computed tube more anatomical and realistic.
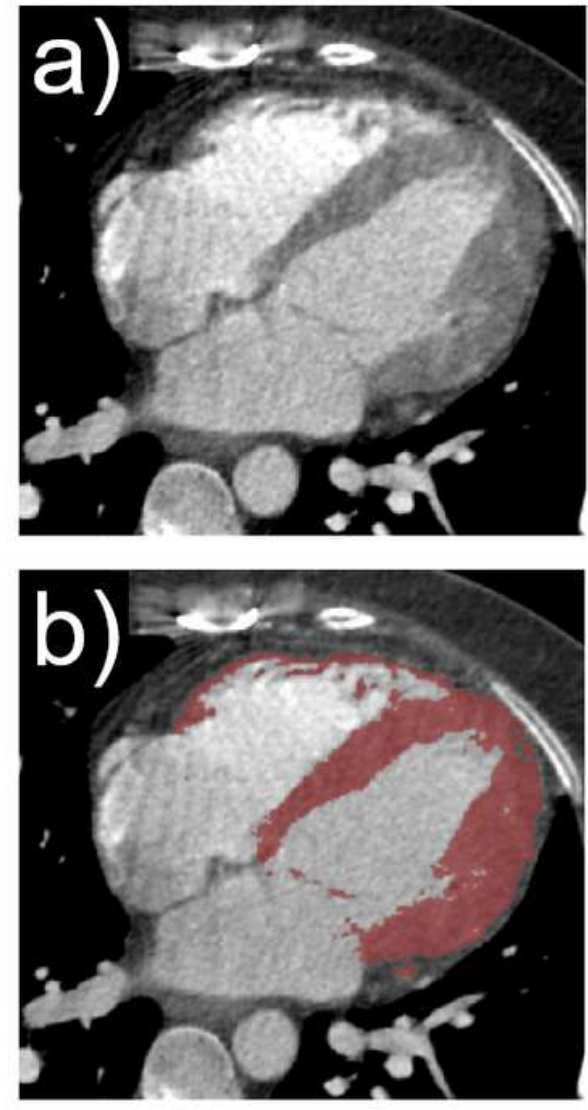
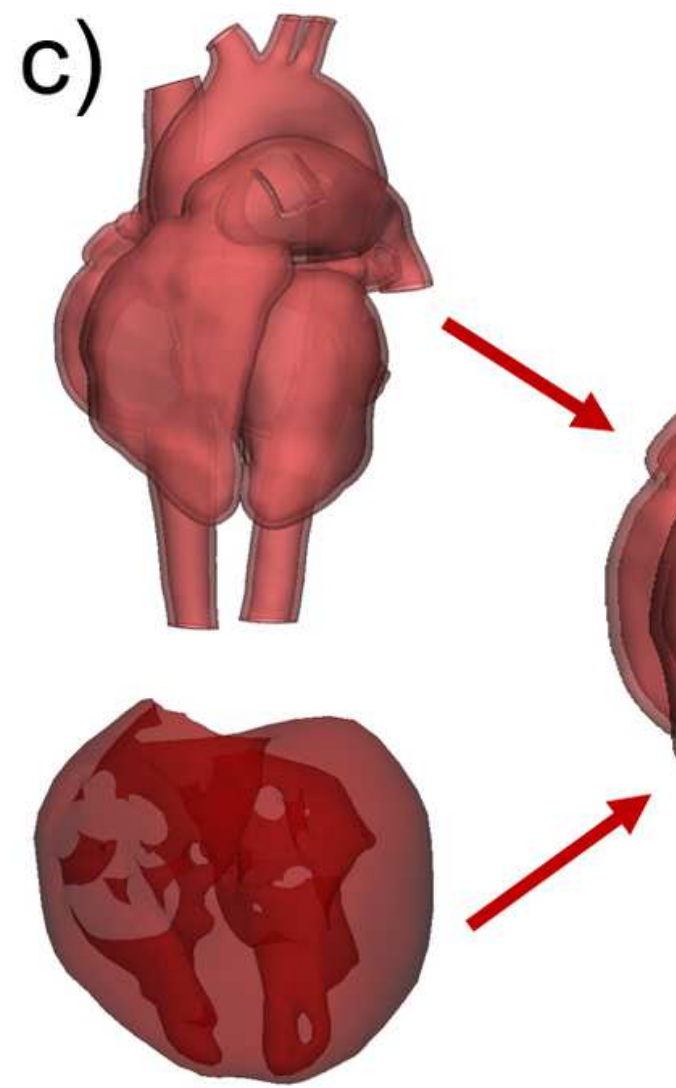

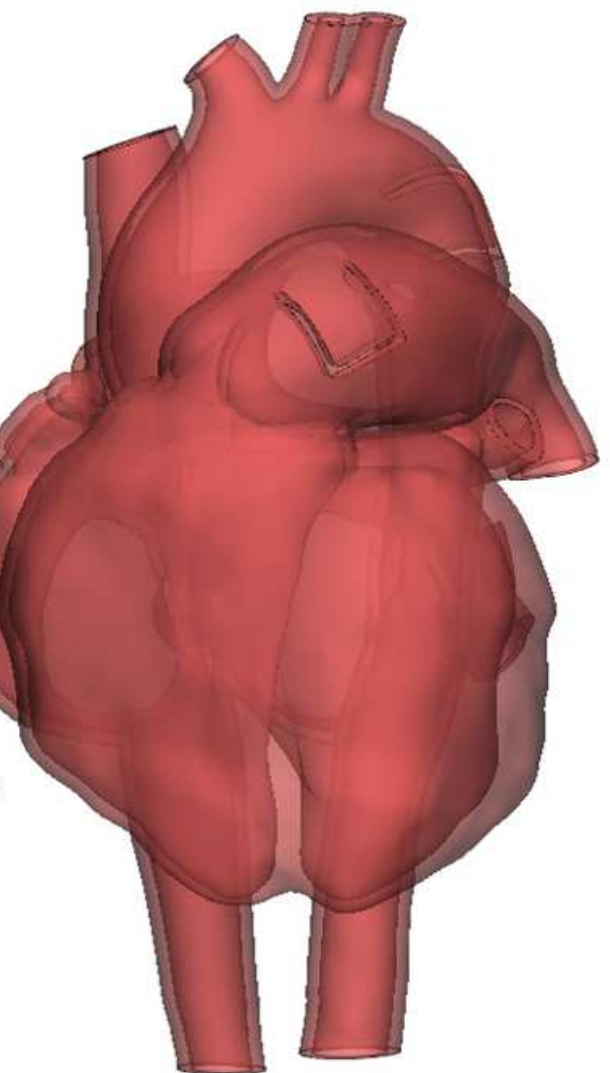

\section{Figure 8}

a) In contrary to the vascular walls the myocardium is visible in CT images. b) Segmentation of the heart muscle is performed by local thresholding techniques. c) Extensive post-processing is required to achieve a smooth outer surface of the myocardium. To maintain the correct anatomical structure of the ventricles 
in the final model the hollow blood volume and the smoothed myocardium are then combined by a series of Boolean operations.

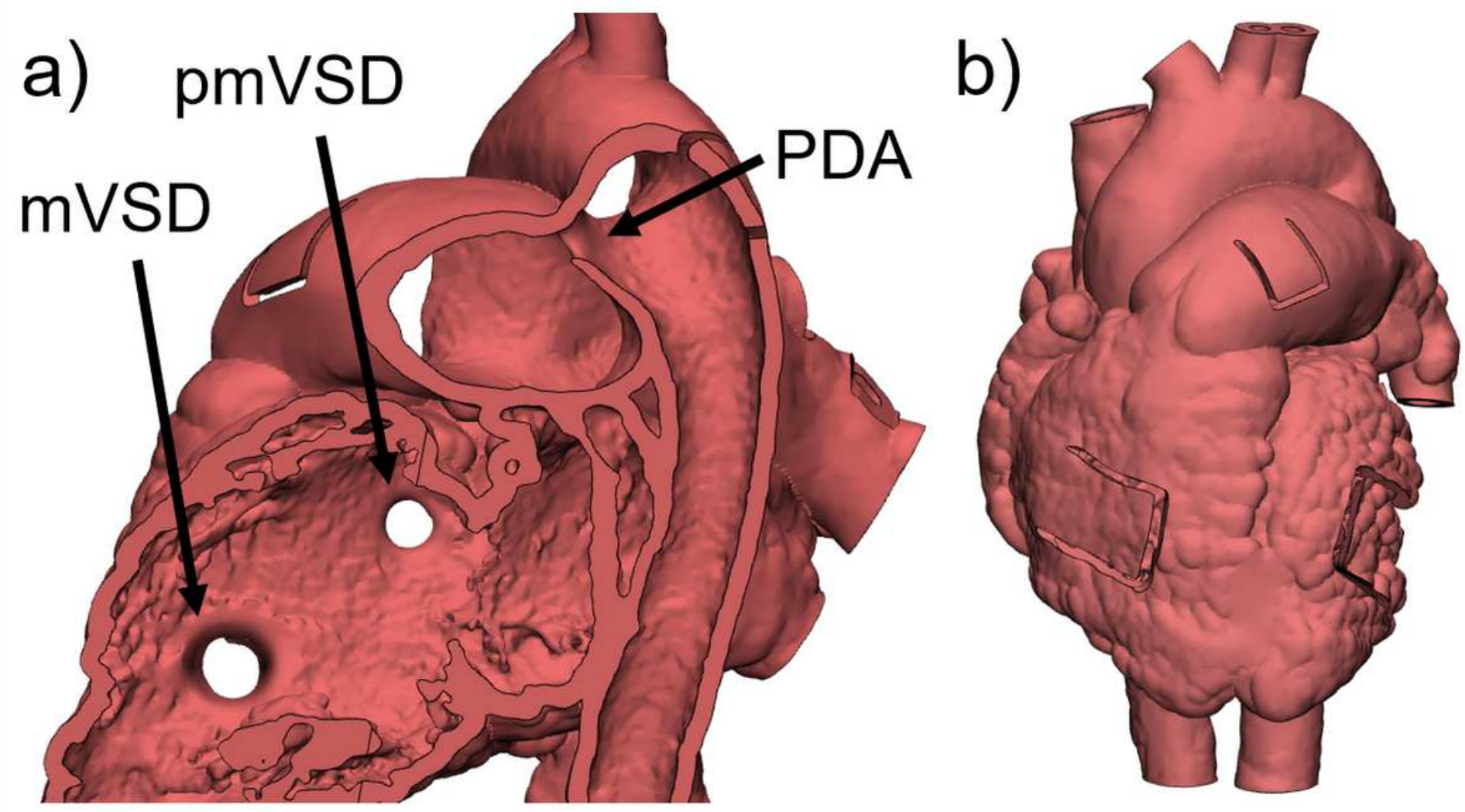

Figure 9

a) For teaching purposes a 3D heart model from a patient with PDA was augmented with two ventricle septum defects, a pmVSD and mVSD respectively. b) Additionally, flap-shaped windows were included into the design to provide insight into the model once 3D printed in a flexible material. 


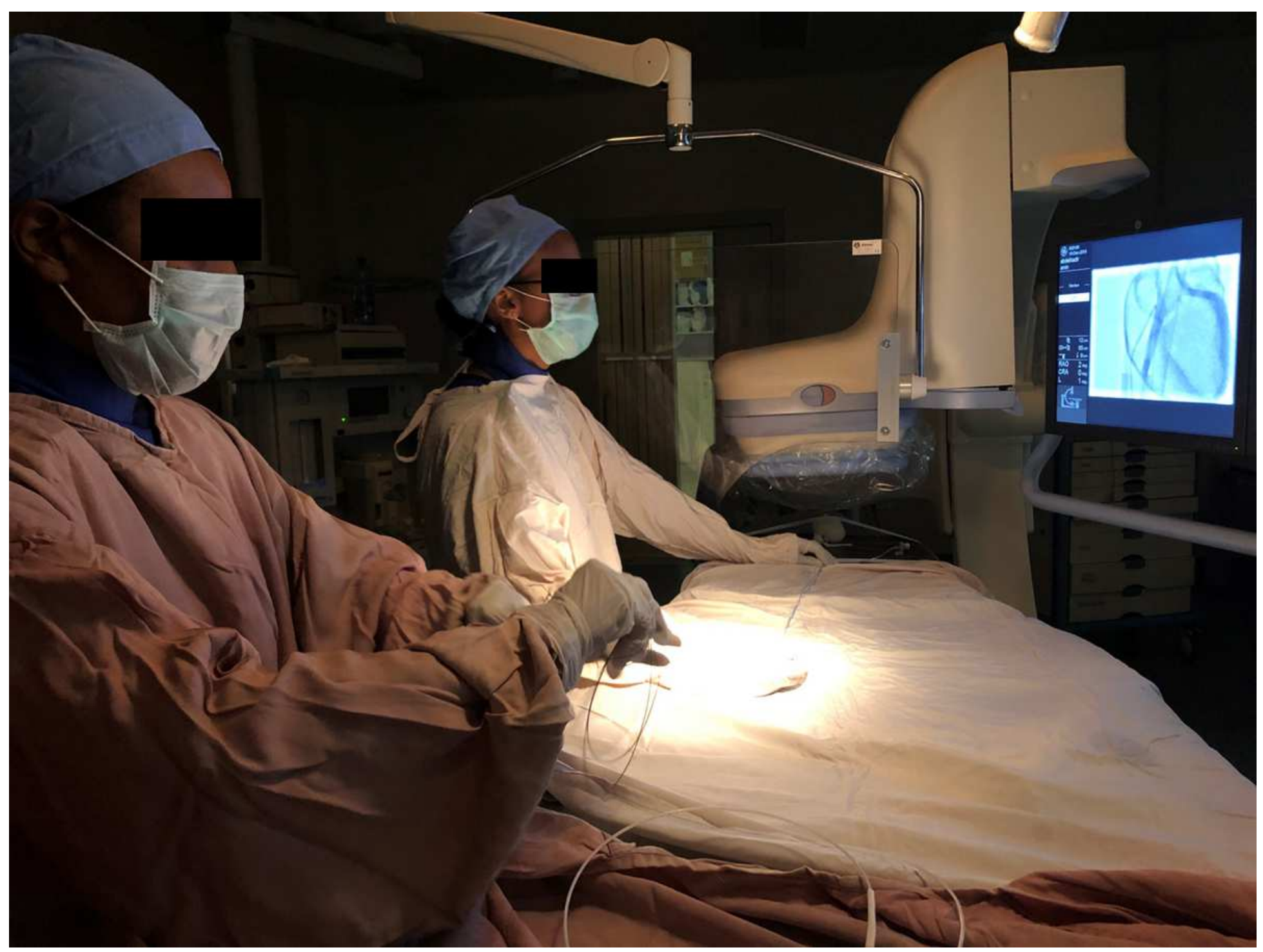

\section{Figure 10}

Within the scope of a partnership project with the Ayder Referral University Hospital Mekelle, Ethiopia, pediatric physicians are trained in diagnostic and interventional pediatric cardiology. The training is performed on 3D printed heart models representing different cases of CHD. The models allow to practice the manipulation of the catheter and the tasks of first and second operator during the procedure before the acquired skills are transferred to real patients. The image shows two pediatric cardiologists training on an infant 3D printed heart model with PS. 


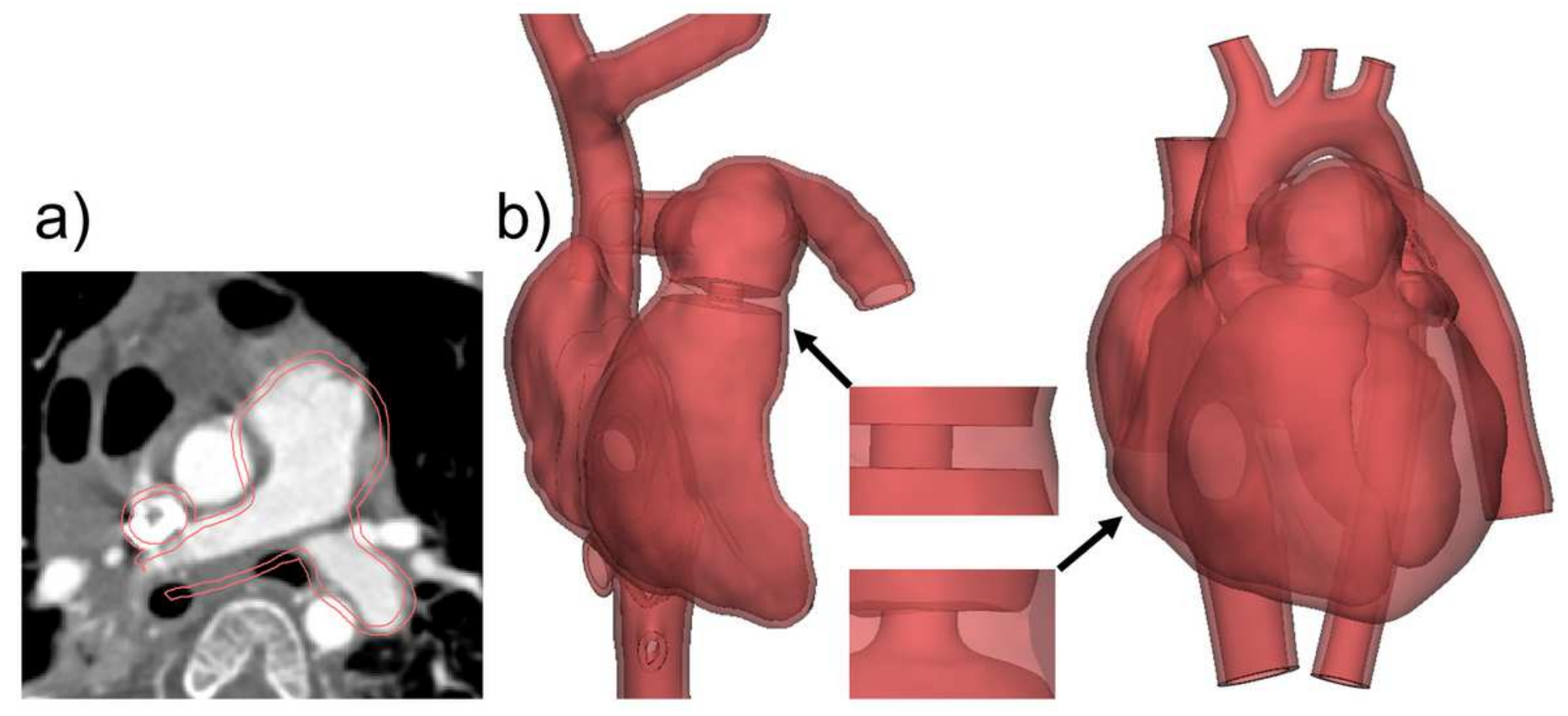

\section{Figure 11}

a) Computer aided design tools allow changing the anatomical structure of $3 \mathrm{D}$ models. The image shows the contours of the adapted and hollowed 3D heart model projected onto the axial image slice of the CT scan it was segmented from. b) The catheter training model was iteratively modified. The first version represented only the right heart with simplified PA. For the latest version the left heart structures and myocardium were added, the pathway towards the pulmonary valve was improved for smooth and realistic catheter manipulation and the shape of the valve was made more realistic.
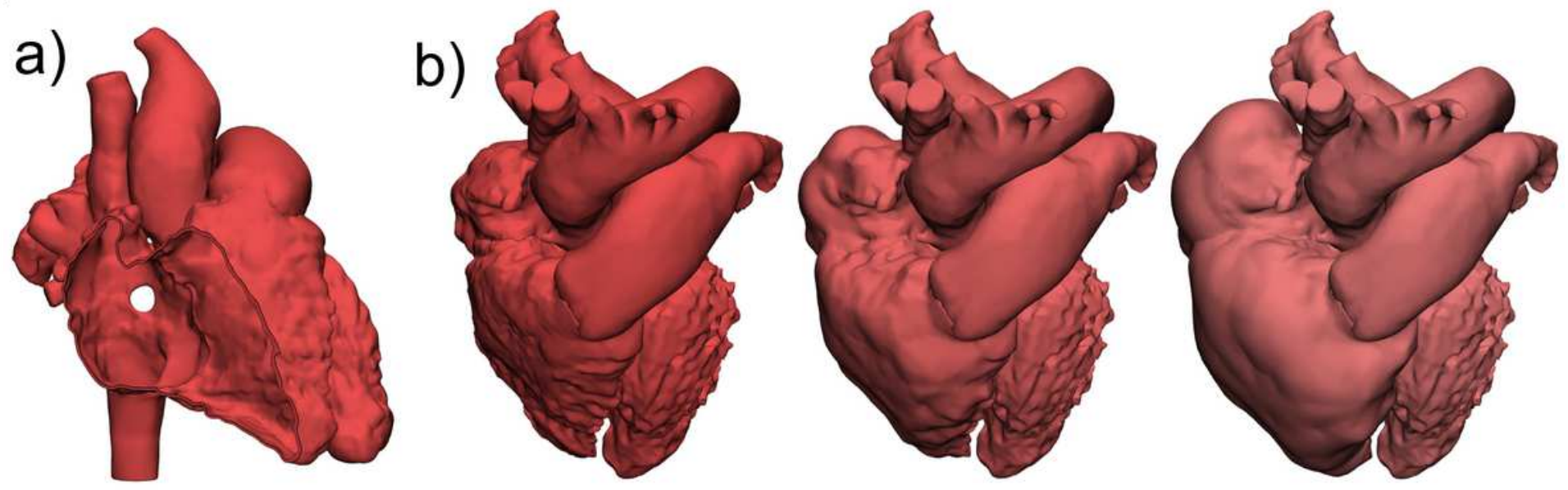

Figure 12

a) A 3D heart model with ASD was engineered based on the dataset of a patient with PDA. b) The hemodynamic effect of an ASD leads to progressive dilation of the right atrium and ventricle due to increased blood volume migrating from the high pressure left heart to the right heart side. This has been illustrated by 3D heart models representing different stages of the disease. 


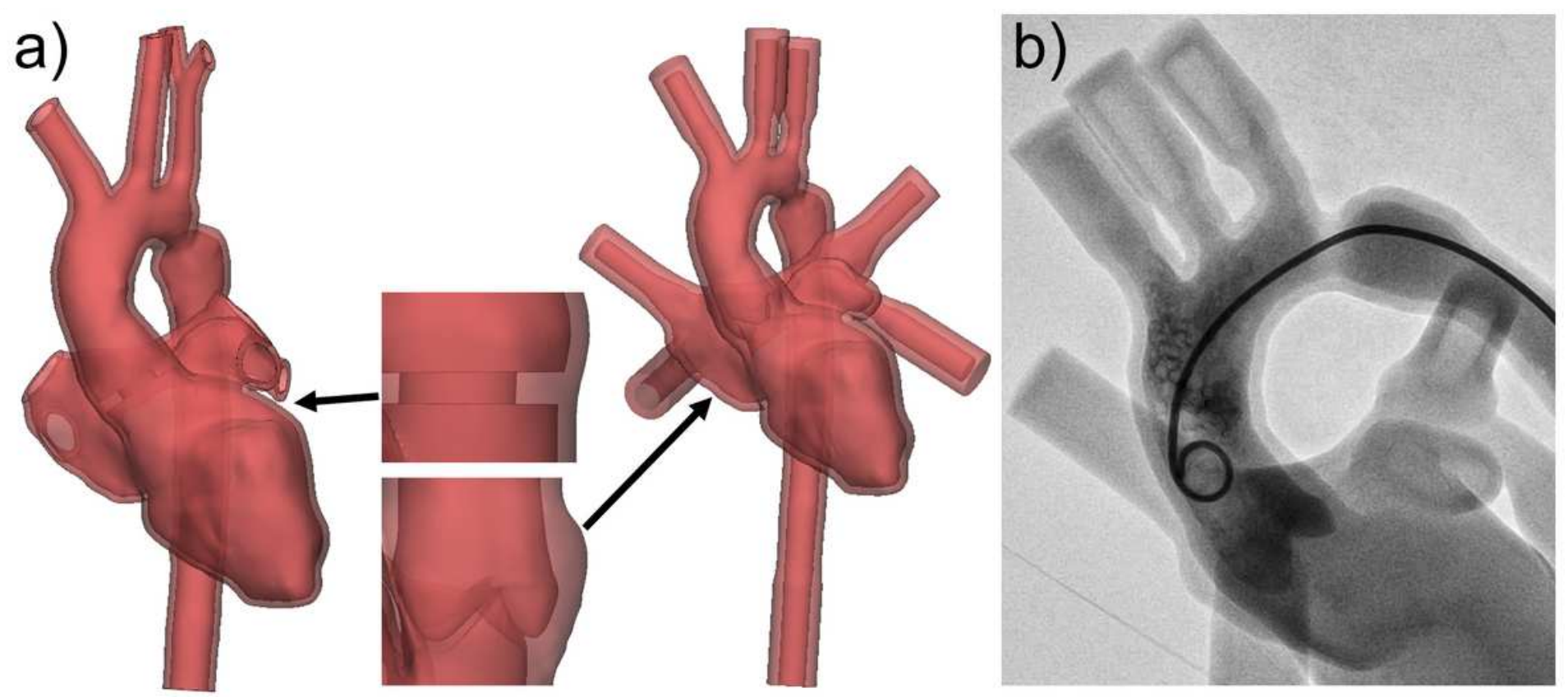

Figure 13

a) The design of a left heart catheter training model with engineered isthmus stenosis and simplified representation of the aortic valve was adapted for connection to a pulsatile flow system. Since the system is filled with water the vessel endings were closed off except for the descending aorta and one pulmonary vein which serve as points of connection to the system. Moreover, a realistic representation of the aortic valve was achieved by replacing the prior design by an engineered STL. b) The water filled beating heart allows advanced catheter training including angiographic imaging. 